Advance Access publication 2019 February 17

GJI Geomagnetism, rock magnetism and paleomagnetism

\title{
Modelling decadal secular variation with only magnetic diffusion
}

\author{
Maurits C. Metman, ${ }^{1}$ Philip W. Livermore ${ }^{\bullet},{ }^{1}$ Jonathan E. Mound ${ }^{1}$ and Ciarán D. Beggan ${ }^{2}$ \\ ${ }^{1}$ School of Earth and Environment, University of Leeds, Leeds LS2 9JT, United Kingdom. E-mail: eemcm@leeds.ac.uk \\ ${ }^{2}$ British Geological Survey, Edinburgh EH14 4AP, United Kingdom
}

Accepted 2019 February 15. Received 2019 February 13; in original form 2018 November 22

\begin{abstract}
S U M M A R Y
Secular variation (SV) of Earth's internal magnetic field is the sum of two contributions, one resulting from core fluid flow and the other from magnetic diffusion. Based on the millenial diffusive timescale of global-scale structures, magnetic diffusion is widely perceived to be too weak to significantly contribute to decadal SV, and indeed is entirely neglected in the commonly adopted end-member of frozen-flux. Such an argument however lacks consideration of radially fine-scaled magnetic structures in the outermost part of the liquid core, whose diffusive timescale is much shorter. Here we consider the opposite end-member model to frozen flux, that of purely diffusive evolution associated with the total absence of fluid flow. Our work is based on a variational formulation, where we seek an optimized full-sphere initial magnetic field structure whose diffusive evolution best fits, over various time windows, a time-dependent magnetic field model. We present models that are regularized based on their magnetic energy, and consider how well they can fit the COV-OBS.x1 ensemble mean using a global error bound based on the standard deviation of the ensemble. With these regularized models, over time periods of up to $30 \mathrm{yr}$, it is possible to fit COV-OBS.x 1 within one standard deviation at all times. For time windows up to $102 \mathrm{yr}$ we show that our models can fit COV-OBS.x 1 when adopting a time-averaged global uncertainty. Our modelling is sensitive only to magnetic structures in approximately the top 10 per cent of the liquid core, and show an increased surface area of reversed flux at depth. The diffusive models recover fundamental characteristics of field evolution including the historical westward drift, the recent acceleration of the North Magnetic Pole and reversed-flux emergence. Based on a global time-averaged residual, our diffusive models fit the evolution of the geomagnetic field comparably, and sometimes better than, frozen-flux models within short time windows.
\end{abstract}

Key words: Dynamo: theories and simulations; Electromagnetic theory; Magnetic field variations through time; Inverse theory; Numerical approximations and analysis.

\section{INTRODUCTION}

The magnetic field generated within the Earth's fluid outer core exhibits continuous change in time over yearly to decadal timescales, termed secular variation (SV). Global geomagnetic field models constructed from ground-based observatories, satellites and other data sources are often expressed in terms of time-dependent Gauss coefficients $\left\{g_{l}^{m}, h_{l}^{m}\right\}$, each of degree $l$ and order $m$, which correspond to a spherical harmonic partitioning of the field; decadal changes of selected spherical harmonic components are shown in Fig. 1.

SV results from two processes within the liquid core: interaction between outer core fluid flow and the magnetic field and magnetic diffusion (e.g. Jackson \& Finlay 2015). The general balance between these two processes is quantified by the magnetic Reynolds number

$R m=\frac{\mathcal{U} \mathcal{L}}{\eta}=\frac{\tau_{d}}{\tau_{u}}$,

with $\mathcal{U}$ and $\mathcal{L}$ characteristic fluid velocity and magnetic length scales, respectively, $\eta$ the magnetic diffusivity and typical timescales for diffusion and advection denoted by $\tau_{\mathrm{d}}$ and $\tau_{\mathrm{u}}$, respectively. Estimates of these quantities $\left(\mathcal{L}=10^{3} \mathrm{~km}, \mathcal{U}=20 \mathrm{~km} \mathrm{yr}^{-1}\right.$ and $\eta=63 \mathrm{~km}^{2} \mathrm{yr}^{-1}$, e.g. Jackson \& Finlay 2015) yield the timescales $\tau_{\mathrm{d}}=16 \mathrm{kyr}$ and $\tau_{\mathrm{u}}=65 \mathrm{yr}$ and $R m \sim 10^{2}$ (where the tilde denotes order of magnitude). The disparity between these timescales implies that for decadal SV diffusion may be neglected, an approximation commonly referred to as that of frozen flux as introduced by Roberts \& Scott (1965). 

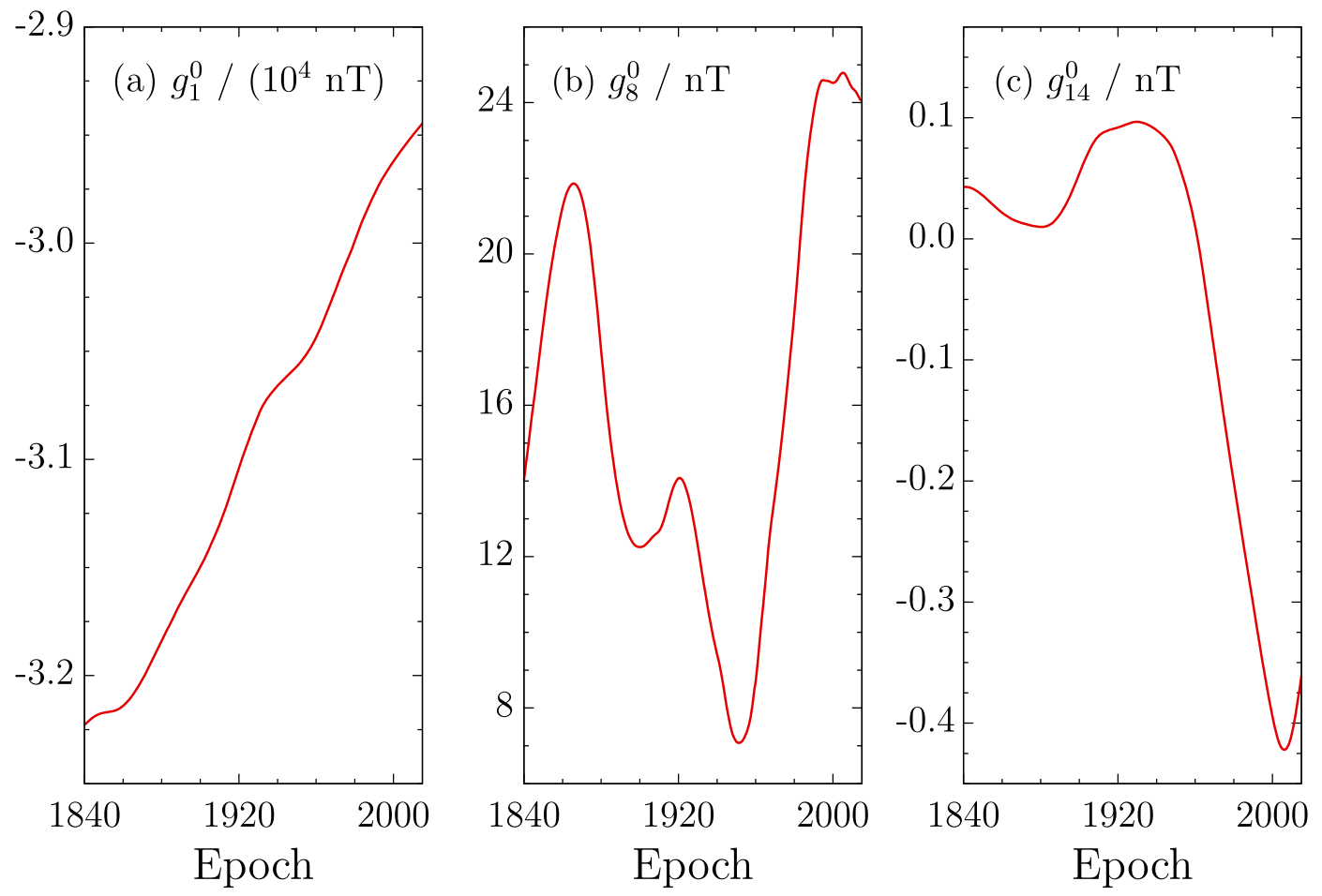

Figure 1. Time-series of the Gauss coefficients $g_{1}^{0}$ (a), $g_{8}^{0}$ (b) and $g_{14}^{0}$ (c) obtained from the mean COV-OBS.x1 field model for the period 1840.0-2015.0 (Gillet et al. 2015a).

This assertion has proven extremely valuable over the past decades. Not only have several authors demonstrated a mathematical consistency between geomagnetic observations and frozen-flux constraints on core field evolution (e.g. Gubbins 1984; Constable et al. 1993; Wardinski \& Lesur 2012), the approximation has also allowed the inversion of SV observations on the Earth's surface and above for core fluid motions along the core-mantle boundary (CMB; e.g. Vestine et al. 1967; Whaler 1980; Bloxham 1988). In addition, frozen flux has been utilized to further constrain the space and time-variability of core fluid flow (Olsen \& Mandea 2008; Aubert 2012; Livermore et al. 2017), to forecast SV over periods of less than a decade (Beggan \& Whaler 2009; Whaler \& Beggan 2015; Bärenzung et al. 2018; Beggan \& Whaler 2018) and to explain length-of-day variations resulting from core-mantle coupling (Jault et al. 1988; Jackson et al. 1993; Gillet et al. 2015b).

Although frozen flux has been shown to be a useful approximation, there is ongoing discussion regarding what part of the observed SV can truly be attributed to magnetic diffusion, which relates to the parameters that define $R m$ (eq. 1). First, typical estimates for $\mathcal{L}$ are based only on horizontal CMB field morphology, and a typical magnetic length scale in the radial direction remains poorly constrained (e.g. Holme 2015), although for the region just below the CMB it has been estimated at several tens to hundreds of km (Amit \& Christensen 2008; Chulliat \& Olsen 2010; Terra-Nova et al. 2016). In light of this, several authors have stressed the inevitable failure of the approximation if magnetic features are indeed of sufficiently small radial scale, for example, due to the concentration of toroidal field beneath the CMB through fluid upwelling, a process known as flux expulsion (Bloxham 1986; Gubbins \& Kelly 1996). Second, the velocity scale $\mathcal{U}$ is usually estimated by directly attributing the observed secular westward drift of equatorial magnetic features (Halley 1692; Finlay \& Jackson 2003) to fluid flow, showing only (in a circular argument) that the frozen-flux approximation is consistent with high $R m$. What this argument does not consider, however, are alternative processes that may explain the westward drift [such as waves (Hide 1966) or diffusion] that would require smaller magnitude flows to explain the residual SV and thus have a smaller associated $\mathrm{Rm}$.

Aside from these theoretical considerations, the importance of diffusion has also been highlighted with numerical simulations of the geodynamo. Such models can yield observable diffusion through flux expulsion (Aubert et al. 2008; Aubert 2014), despite the fact that these models are typically characterized by Earth-like $R m$ (Christensen et al. 2010). Furthermore, the special geodynamo case of steady core fluid motion has been demonstrated to be incompatible with frozen flux (Gubbins \& Kelly 1996; Love 1999). More recently, Barrois et al. (2017) employed statistics derived from geodynamo simulations to demonstrate that even on short timescales diffusion contributes up to approximately 10 per cent of the overall $\mathrm{SV}$ at the $\mathrm{CMB}$, although separating this diffusive signal from errors related to finite horizontal resolution remains non-trivial (Barrois et al. 2018).

Local failure of frozen flux has also been inferred from observations of the core magnetic field, using the constraint that without diffusion the total magnetic flux through patches bounded by null-flux curves should remain steady (Backus \& Bullard 1968). For instance, recent rapid change of the North Magnetic Pole (NMP) and of the radial magnetic field under St. Helena have been attributed to flux expulsion (Chulliat et al. 2010; Chulliat \& Olsen 2010). Gubbins (1996) made an initial attempt to invert for core motion with the explicit inclusion of diffusion and estimated a considerable radial gradient of toroidal field of $20 \mathrm{nT} \mathrm{m}^{-1}$ beneath the South Atlantic, sufficient to explain the local intensification of magnetic flux of $500 \mathrm{MWb}$. However, it remains difficult to unambiguously detect such expulsion patches and hence a 
signature of magnetic diffusion in the core (Amit 2014). Furthermore, it is challenging to distinguish magnetic diffusion from energy transfer between unresolved small-scale flow and the observed large-scale magnetic field (Roberts \& Glatzmaier 2000).

In this paper, we will test whether magnetic diffusion is capable of generating decadal SV that matches the observation-based model COV-OBS.x1 (Gillet et al. 2015a). To do this, we use a variational formulation, where we seek the 3-D structure of an initial magnetic field whose subsequent evolution best explains SV over a defined time window. Variational data assimilation has been implemented successfully in several nonlinear geodynamo models (Fournier et al. 2007; Li et al. 2014) but here, owing to the lack of any coupling through the absent flow, the optimal initial field can be found in a particularly simple way as a solution to a linear system. We consider various time windows to assess whether a purely diffusive model is consistent with SV on yearly, decadal and centennial timescales. This approach allows us to explore a new end-member model of the SV, that of pure diffusion, and compare it against models of the more traditional frozen-flux type. Our purely diffusive model is not intended to fully represent the mechanism responsible for SV, but rather to test whether it is sufficient to reconstruct the observed SV and to probe the field below the CMB using the inherent assumptions of the model. While the formalism by Gubbins (1996) permits diffusion as a correction term for frozen flux and could be utilized to jointly invert observed field evolution for the frozen-flux and diffusive contributions to SV, we present here a new scheme that considers diffusion as the sole contribution to SV.

This work is structured as follows: we first describe in Section 2 how we optimize the initial full-sphere magnetic field to match SV through its diffusive evolution; the resultant diffusive models along with their general and local characteristics are presented in Section 3; Section 4 discusses the geophysical implications of our results. Four appendices describe technical details including depth-sensitivity and numerical convergence.

\section{METHODS}

In this section we present our variational formalism for a diffusing 3-D magnetic field. Assuming that the solid inner core has the same electrical conductivity as the liquid core, the same diffusion equation is obeyed everywhere in the core by the magnetic field and we therefore seek full-sphere solutions, which are also therefore valid in a spherical shell. We begin by briefly introducing the associated forward problem and its canonical solutions: the decay modes. These functions are used in Appendix A to determine the resolving power of our methodology as a function of depth. We also present an alternative representation of the forward model using Galerkin polynomials, which turns out to be numerically much better conditioned than that based on decay modes. Finally, we consider a means of regularizing the inverse solution by penalizing the total magnetic energy.

\subsection{Decay modes}

Here, a set of analytical (forward) solutions to the (dimensional) magnetic diffusion equation are given (Gubbins \& Roberts 1987). If we were provided with the structure of the magnetic field throughout the entire core, these solutions would describe the temporal evolution of that field in the absence of fluid flow. We start with the 3-D magnetic diffusion equation for uniform magnetic diffusivity, which reads

$\left(\frac{\partial}{\partial t}-\eta \nabla^{2}\right) \mathbf{B}=\mathbf{0}$

where $t$ is time and $\mathbf{B}$ denotes the core field. Because the field is solenoidal, it may be partitioned into its toroidal and poloidal parts:

$\mathbf{B}=\mathbf{B}_{\mathrm{T}}+\mathbf{B}_{\mathrm{S}}=\nabla \times(T \mathbf{r})+\nabla \times \nabla \times(S \mathbf{r})$

with $\mathbf{B}_{\mathrm{T}}$ and $\mathbf{B}_{\mathrm{S}}$ the toroidal and poloidal field, respectively, and $T$ and $S$ their respective defining potentials. Applying this decomposition simplifies the diffusion problem to the scalar equations

$\left(\frac{\partial}{\partial t}-\eta \nabla^{2}\right) T=\left(\frac{\partial}{\partial t}-\eta \nabla^{2}\right) S=0$.

Both of these equations may be solved to obtain a diffusive description for all vector components of the field. However, the toroidal field inside the core is unobservable (the field outside the core is purely poloidal) and we therefore restrict attention to the poloidal diffusion problem by setting $T=0$. The scalar $S$ is then expanded in real-valued Schmidt quasi-normalized spherical harmonics $Y_{\alpha}(\theta, \phi)(\theta$ and $\phi$ denote colatitude and longitude, respectively), the index $\alpha$ corresponding to the harmonic of degree $1 \leq l_{\alpha} \leq L$ for some $L$ and order $0 \leq m_{\alpha} \leq l_{\alpha}$, which has either azimuthal sine or cosine dependence. This representation then requires

$\left(\frac{\partial}{\partial t}-\eta D_{l_{\alpha}}^{2}\right) r s_{\alpha}(r, t)=0$

with $r$ radius, $s_{\alpha}$ the spherical harmonic coefficients of $S$ and where we have defined the operator

$D_{l_{\alpha}}^{2} f(r)=\left(\frac{\partial^{2}}{\partial r^{2}}-\frac{l_{\alpha}\left(l_{\alpha}+1\right)}{r^{2}}\right) f(r)$. 

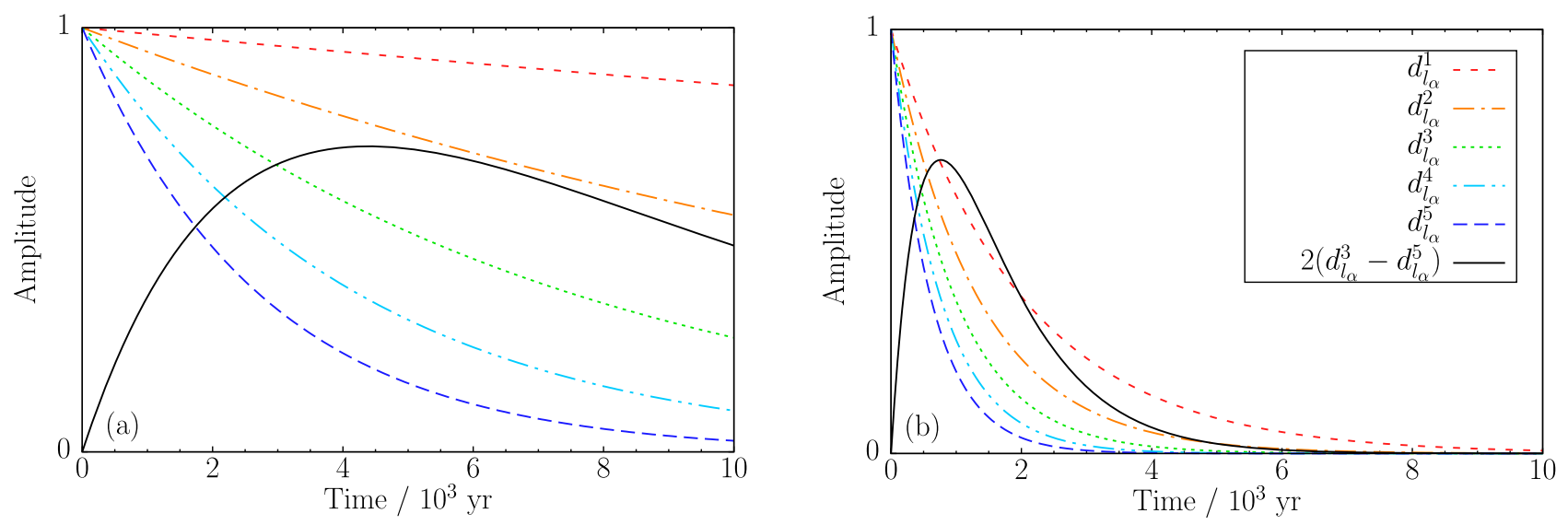

Figure 2. Time dependence of the first five decay modes $d_{l_{\alpha}}^{n}$ for (a) $l_{\alpha}=1$ and (b) $l_{\alpha}=14$, normalized to have initial positive unit amplitude. While a single mode always decays monotonically, a linear combination of these functions may exhibit transient strengthening (solid curve).

This equation has the solution

$$
\begin{aligned}
s_{\alpha}(r, t) & =\sum_{n=1}^{N} q_{\alpha}^{n} d_{l_{\alpha}}^{n}(r, t), \\
& =\sum_{n=1}^{N} q_{\alpha}^{n} j_{l_{\alpha}}\left(\frac{k_{l_{\alpha}}^{n} r}{c}\right) \exp \left[-\eta\left(\frac{k_{l_{\alpha}}^{n}}{c}\right)^{2}\left(t-t_{0}\right)\right],
\end{aligned}
$$

where $d_{l_{\alpha}}^{n}(r, t)$ denotes the $n$th decay mode of degree $l_{\alpha}$, the $q_{\alpha}^{n}$ a set of constants, $j_{l_{\alpha}}(z)$ the spherical Bessel function of the first kind, $k_{l_{\alpha}}^{n}$ the $n$th root of $j_{l_{\alpha}+1}(z)$ and $c$ the core radius. The initial state is given by the expression evaluated at the initial time $t_{0}$. The decay modes form an orthogonal set in terms of an all-space energy norm (Backus 1958). This solution allows us to express the radial field $B_{\mathrm{r}}$ up to degree $L$ satisfying the diffusion equation as

$$
\begin{aligned}
B_{\mathrm{r}}(\mathbf{r}, t) & =\frac{1}{r} \sum_{\alpha=1}^{L(L+2)} l_{\alpha}\left(l_{\alpha}+1\right) s_{\alpha}(r, t) Y_{\alpha}(\theta, \phi), \\
& =\frac{1}{r} \sum_{\alpha=1}^{L(L+2)} \sum_{n=1}^{N} q_{\alpha}^{n} l_{\alpha}\left(l_{\alpha}+1\right) j_{l_{\alpha}}\left(\frac{k_{l_{\alpha}}^{n} r}{c}\right) \exp \left[-\eta\left(\frac{k_{l_{\alpha}}^{n}}{c}\right)^{2}\left(t-t_{0}\right)\right] Y_{\alpha}(\theta, \phi) .
\end{aligned}
$$

Using this decay mode expansion, we may already describe our following inverse approach qualitatively. Consider, for example, Fig. 2 showing the time dependence of the first five modes for $l_{\alpha}=1$ (a) and $l_{\alpha}=14$ (b), where their initial amplitude has been normalized to unity. It can readily be seen that all individual modes decay monotonically with time, and that their respective rate of decay increases with $l_{\alpha}$ and $n$. However, a linear combination of modes need not always decay, as is the case for the difference $d_{l_{\alpha}}^{3}-d_{l_{\alpha}}^{5}$ (solid black curve), which exhibits transient growth (e.g. Livermore \& Jackson 2006). Although the modes are formally orthogonal in a global sense, they are not orthogonal when evaluated at a single value of $r=c$ and thus transient effects can readily occur. In the same way, there may exist a combination of modes that describes an initial field configuration whose evolution matches the observed SV (which shows in general both transient decay and growth) over some time window.

We also use the decay modes a priori to estimate the sensitivity of the CMB field evolution to magnetic diffusion within different regions of the core. It may be expected that the core is not sampled uniformly; we consider diffusion only over time intervals of decades to centuries, and bearing in mind the slow $16 \mathrm{kyr}$ timescale of global modes, the diffusive evolution of deep magnetic structures may not have a signature in the CMB SV within such time intervals. In Appendix A, we show that for pure diffusion the CMB field evolution can only constrain the magnetic structure within a region close to the CMB. This layer spans approximately the top $80 \mathrm{~km}$ when one decade of diffusion is considered (Fig. A2a); for $175 \mathrm{yr}$, which matches the full COV-OBS.x1 time window, this region extends to roughly the upper $400 \mathrm{~km}$ (Fig. A2b). Therefore, even though we shall seek an optimal full-sphere magnetic structure, structures below the sensitivity depth should not be interpreted geophysically.

\subsection{A Galerkin discretization}

While the decay modes solve the magnetic diffusion equation exactly, the series in eq. (8) is characterized by slow (algebraic) convergence in $N$ (e.g. Boyd 2000), associated with poor numerical conditioning of the inverse problem. In the following, as an expedient alternative, we solve the radial diffusion problem (eq. 5) with a Galerkin polynomial basis set in radius, yielding faster (spectral) convergence and much 

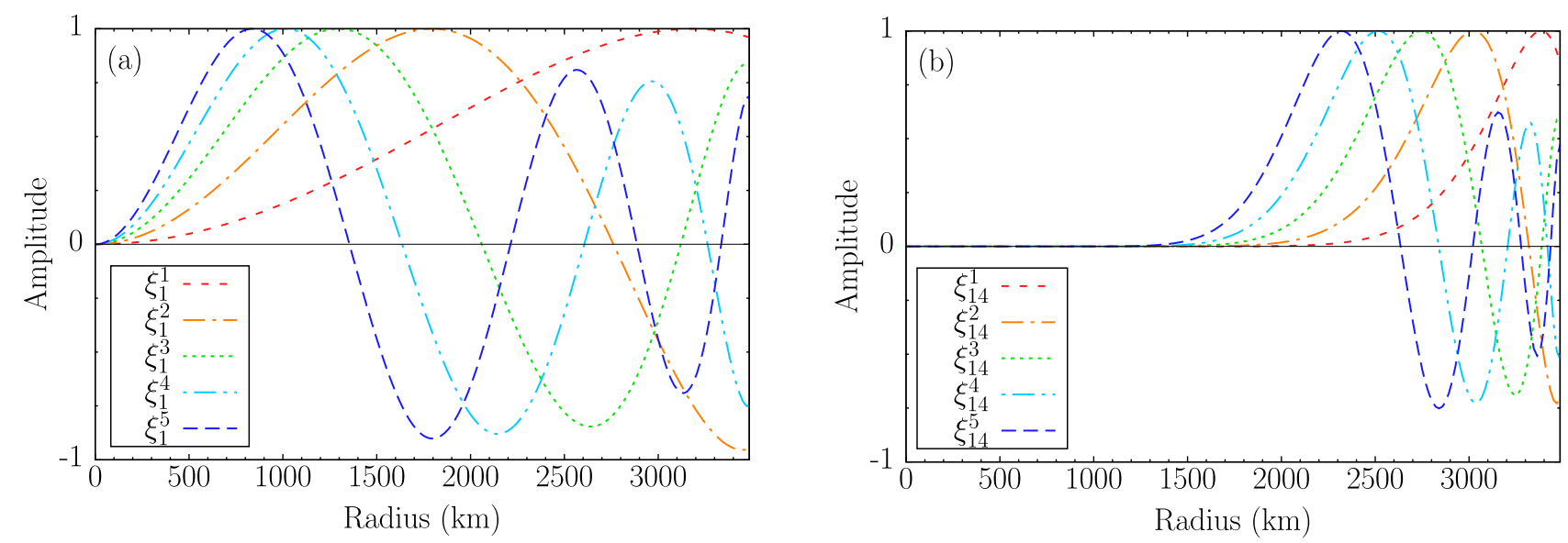

Figure 3. The Jacobi polynomials $\xi_{l_{\alpha}}^{n}$ as a function of radius, for $l_{\alpha}=1$ (a) and $l_{\alpha}=14$ (b), here normalized such that their extremum is of positive unit amplitude.

better conditioning. To do so, we approximate

$r s_{\alpha}(r, t)=\sum_{n=1}^{N} \xi_{\alpha}^{n}(r) \varphi_{\alpha}^{n}(t) q_{\alpha}^{n}$,

with $\varphi_{\alpha}^{n}(0)=1$. We choose the radially dependent basis functions to be built from weighted Jacobi polynomials (Livermore 2010) of the form

$\xi_{\alpha}^{n}(r)=A_{\alpha}^{n} \psi_{\alpha}^{n}(r / c)$

where $A_{\alpha}^{n}$ is a normalization constant and

$\psi_{\alpha}^{n}(r)=r^{l_{\alpha}+1}\left[n(2 l+2 n-1) P_{n}^{\left(0, l_{\alpha}+1 / 2\right)}\left(2 r^{2}-1\right)-(n+1)(2 l+2 n+1) P_{n-1}^{\left(0, l_{\alpha}+1 / 2\right)}\left(2 r^{2}-1\right)\right]$,

with $P_{n}^{(\alpha, \beta)}(z)$ a Jacobi polynomial. The poloidal vector modes $\mathbf{B}_{\alpha}^{n}=\nabla \times \nabla \times\left(\xi_{\alpha}^{n} Y_{\alpha} \hat{\mathbf{r}}\right)$ have the property of orthogonality over all space (Chen et al. 2018; Li et al. 2018) through the integral (see also Appendix D)

$$
\begin{aligned}
& \int_{R^{3}} \mathbf{B}_{\alpha}^{n} \cdot \mathbf{B}_{\beta}^{p} \mathrm{~d} V=\frac{4 \pi l_{\alpha}\left(l_{\alpha}+1\right)}{2 l_{\alpha}+1}\left[\int_{0}^{c}\left(\frac{\partial \xi_{\alpha}^{n}}{\partial r}\right)\left(\frac{\partial \xi_{\beta}^{p}}{\partial r}\right)+\frac{l_{\alpha}\left(l_{\alpha}+1\right)}{r^{2}} \xi_{\alpha}^{n} \xi_{\beta}^{p} \mathrm{~d} r+\frac{l_{\alpha}}{c} \xi_{\alpha}^{n}(c) \xi_{\beta}^{p}(c)\right] \\
& :=\left\langle\xi_{\alpha}^{n}, \xi_{\beta}^{p}\right\rangle_{l_{\alpha}},
\end{aligned}
$$

which may be used to find the constant $A_{\alpha}^{n}$. Such orthogonality is identical to that satisfied by the decay modes, but the Galerkin modes are much better numerically conditioned as they show asymptotic spectral convergence. The radial dependence of these functions is visualized in Fig. 3, which are not only regular at the origin, but also obey the matching conditions appropriate to an external potential field.

Through substitution of the approximation (eq. 9) in the radial diffusion equation (eq. 5), and by projecting the result on $\xi_{\alpha}^{j}(r)$ using eq. (), we obtain the system of equations:

$\frac{\partial}{\partial t} \boldsymbol{\varphi}_{\alpha}=\eta \mathbf{H}_{\alpha} \boldsymbol{\varphi}_{\alpha}$

where $\varphi_{\alpha}:=\left(\varphi_{\alpha}^{1}, \varphi_{\alpha}^{2}, \ldots, \varphi_{\alpha}^{N}\right)^{T}$ is the time-dependent solution for a given $\alpha$ and $H_{\alpha, i j}:=\left\langle D_{l_{\alpha}}^{2}\left[\xi_{\alpha}^{i}\right] \text {, } \xi_{\alpha}^{j}\right\rangle_{l_{\alpha}}$. The total solution, exact within the truncation specified, can be written

$r s_{\alpha}(r, t)=\boldsymbol{\xi}_{\alpha}^{T}(r) \operatorname{expm}\left[\eta \mathbf{H}_{\alpha}\left(t-t_{0}\right)\right] \mathbf{q}_{\alpha}$,

where expm $[\cdot]$ denotes the matrix exponential. When $t=t_{0}$, the given matrix exponential is the identity matrix, in which case one recovers the initial magnetic structure. Note also that for the particular case of using the decay modes in place of the Galerkin polynomials, the matrix $\mathbf{H}_{\alpha}$ and so expm[$\left[\mathbf{H}_{\alpha}\right]$ are diagonal, and so the above representation is equivalent to the solution given in eq. (7). 


\subsection{Unregularized inverse strategy}

\subsubsection{A least-squares variational analysis}

With this discretization of the diffusive forward problem, we are now in a position to define the variational scheme. Accordingly, we consider the objective function

$R_{\text {unreg }}=\int_{T} \int_{\mathrm{CMB}}\left(B_{\mathrm{r}}^{\mathrm{obs}}(t)-\hat{B}_{\mathrm{r}}(t)\right)^{2} \mathrm{~d} S \mathrm{~d} t$

where the time period is $T=\left[t_{0}, t_{e}\right], B_{\mathrm{r}}^{\text {obs }}$ is the radial field prescribed by a time-dependent geomagnetic field model, and $\hat{B}_{\mathrm{r}}(t)$ denotes the radial component of our modelled field. We seek an initial structure of the magnetic field that minimizes $R_{\text {unreg. }}$.

Discretizing the temporal integrals and expressing $R_{\text {unreg }}$ in terms of the model coefficients gives the reduced form

$R_{\text {unreg }}=(\mathbf{g}-\mathbf{D} \hat{\mathbf{q}})^{T} \mathbf{W}(\mathbf{g}-\mathbf{D} \hat{\mathbf{q}})$,

with $\mathbf{g}$ and $\hat{\mathbf{q}}$ vectors containing all Gauss coefficients (which are known) evaluated at a set of time points and all model coefficients (which are to be found), respectively, the matrix D a blockwise-diagonal (one block per spherical harmonic mode) forward mapping describing purely diffusive SV, and $\mathbf{W}$ a diagonal weighting matrix related to the scheme of numerical time integration. Adopting decay modes in place of Galerkin polynomials results in a comparable form but with different $\mathbf{D}$. For the derivation and exact definition of these quantities, the reader is referred to Appendix B. Key here is the fact that the objective function depends only quadratically on $\hat{\mathbf{q}}$, in contrast to much more complex schemes that include nonlinear feedback from the core flow (e.g. Li et al. 2014). Taking variations to find the minimum of $R_{\text {unreg, with respect }}$ to $\hat{\mathbf{q}}$, then directly gives the optimal set of coefficients

$\hat{\mathbf{q}}=\left(\mathbf{D}^{T} \mathbf{W D}\right)^{-1} \mathbf{D}^{T} \mathbf{W g}$.

In this paper we will consider only using this variational technique with the geomagnetic field model given by the mean of the COVOBS.x1 ensemble (Gillet et al. 2015a). This model of spherical harmonic degree $L=14$ spans 175yr (1840.0-2015.0) that is sufficient to cover our range of time window lengths and extends (almost) to the present day using modern satellite data.

It is worthwhile briefly considering our inversion scheme in the landscape of other mathematical inverse problems associated with diffusion. A well-known example is the backwards heat conduction problem (e.g. Miranker 1961) in which complete knowledge of a temperature profile is assumed at some final time $t=t_{\mathrm{e}}$, and the task is to find the initial temperature profile at $t=t_{0}$ that evolves through heat diffusion (conduction) to match the final state; such a problem is, in general, tractable but ill-posed. The problem that we consider here is quite different: as described above, the structure of the evolving magnetic field is known only at $r=c$ (rather than everywhere), but for all times in $\left[t_{0}, t_{\mathrm{e}}\right]$ (rather than at a single time). We have therefore traded complete information of structure at a single time for partial structural information at all times. A solution of the backwards conduction problem requires two boundary conditions, as it is a second-order equation like the magnetic diffusion equation. In our case we also supply two sets of information at the boundary. First we constrain the poloidal scalar through matching to the field model. Second, we constrain the radial derivative of the poloidal scalar by virtue of the matching condition associated with the electrically insulating mantle at $r=c$. Our problem is therefore fully specified and optimal solutions exist, although we may not be able to fit the data exactly $\left(R_{\text {unreg }}>0\right.$, see e.g. Appendix C).

\subsubsection{Matrix conditioning and choice of radial basis functions}

In order for our inverse approach to be viable, it is necessary that the solution of eq. (18) is numerically realizable. Of particular concern is whether the numerical inverse of the weighted Gramian matrix $\mathbf{D}^{T} \mathbf{W D}$ is computationally tractable, which can be quantified by its condition number. As an approximate rule of thumb, if $\mathbf{D}^{T} \mathbf{W D}$ has a condition number $10^{a}$, the number of inaccurate trailing digits of $\hat{\mathbf{q}}$ is $a$ when the solution is expressed numerically with floating-point representation. Thus in standard double-precision (about 16 digits), with a condition number of $10^{12}, \hat{\mathbf{q}}$ can be expected correct to about four significant figures; matrices with condition numbers in excess of $10^{16}$ or so cannot be inverted in double precision.

Fig. 4 shows the condition numbers (measured in the standard 2-norm) for the diagonal blocks of $\mathbf{D}$ associated with $l_{\alpha}=1$ and $l_{\alpha}=14$, representing the extreme cases, as a function of the number of basis functions (either polynomial or decay modes). Here, we used 201 time points (this ensures a numerically converged solution, see Appendix C), and sextuple (256-digit) precision in place of the more commonly used double (16-digit) precision to ensure that all calculations shown were accurate. The condition numbers, many of which are far in excess of $10^{16}$, highlight the ill-posedness of the inverse problem. Physically, this may be understood by the difficulty of constraining the magnitude of initially fine scales because they diffuse very rapidly: this is a problem for both the decay mode and Jacobi polynomial representations. In practice, the large condition numbers mean that in double precision the radial resolution available for this method is extremely limited.

However, there are two ways in which we can mitigate these effects. First, it should be noted that the Galerkin scheme has a much lower condition number than the decay modes for fixed $N$, an effect particularly notable for block matrices of high spherical harmonic degree. This means that for any given condition number threshold the Galerkin method allows more radial modes and therefore increased resolution. The better conditioning achieved with the Galerkin method therefore motivates us to apply it to our inverse approach. Second, we can use very 


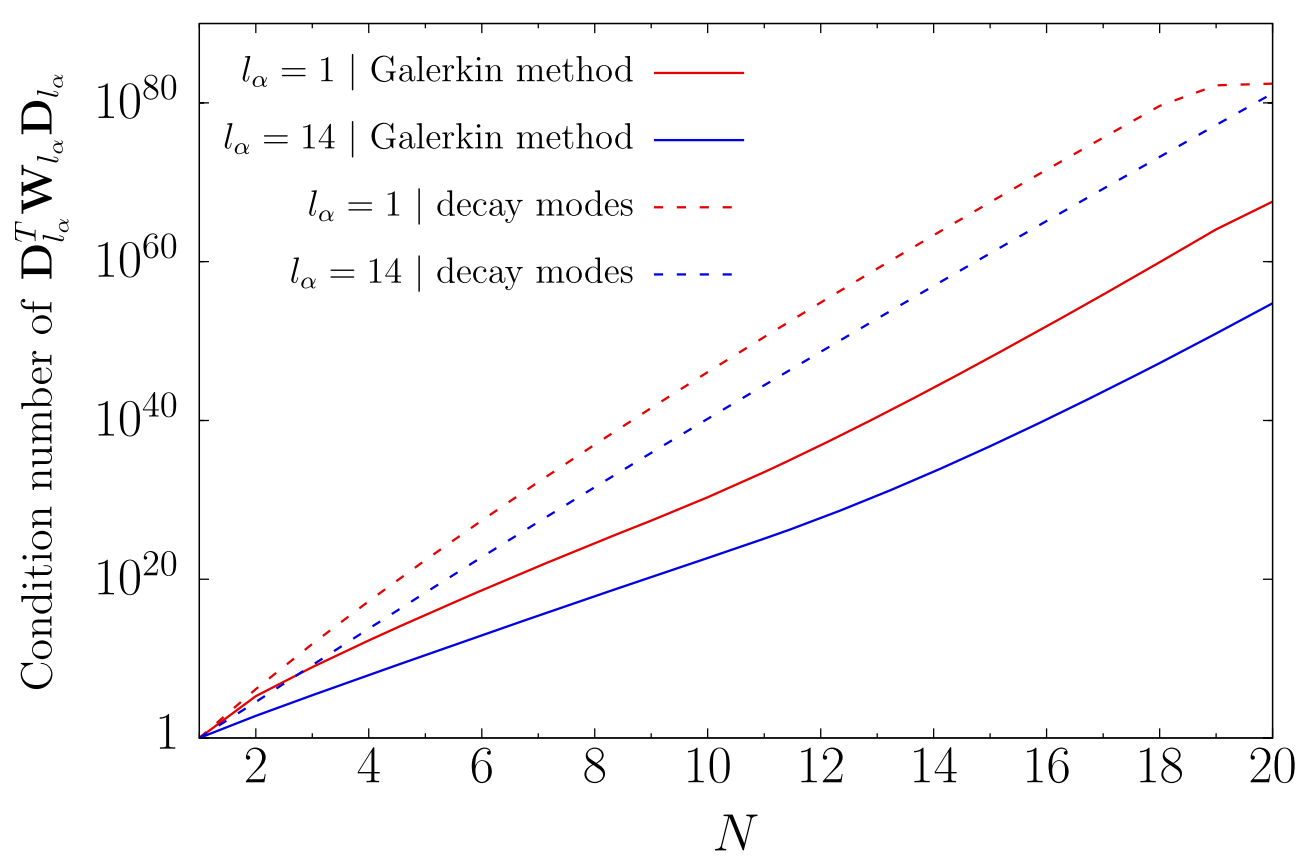

Figure 4. The condition number of the weighted Gramian block matrices, for $l_{\alpha}=1$ (red) and $l_{\alpha}=14$ (blue), as a function of $N$, the number of basis functions used and using either decay modes (dashed) or the Galerkin polynomials (solid).

high precision in our calculations (we have used up to 256 digits using the symbolic toolbox for Matlab), making possible a resolution of 30 radial polynomial modes (with typical condition numbers of $10^{95}$ and $10^{70}$ for the decay modes and Galerkin polynomials, respectively).

\subsection{Regularized inverse strategy}

\subsubsection{A penalized variational approach}

Minimizing an objective function of the form of $R_{\text {unreg }}$ (eq. 16) ensures an optimal fit to SV without consideration of the spatial complexity of the initial optimal magnetic field. It may therefore be unsurprising that the initial $\left(t=t_{0}\right)$ magnetic structures can show large spatial fluctuations in amplitude. For example, if we consider the optimization over the time window 2005.0-2015.0, the associated initial field over the upper $80 \mathrm{~km}$ of the core (its sensitivity depth: the field below this depth is likely poorly resolved, see Appendix A), we find a root-mean-square (RMS) average $|\mathbf{B}|$ of about $10^{25} \mathrm{mT}$. Similarly, for the period 1840.0-2015.0, the optimal model has an RMS field strength in excess of $10^{29} \mathrm{mT}$ over its sensitivity depth of $400 \mathrm{~km}$. Such huge amplitudes cannot be reconciled with the RMS field strength for the core, which has been estimated at 2.5 to $4 \mathrm{mT}$ (Buffett 2010; Gillet et al. 2010). It is likely that the ill-posedness of the unregularized inverse diffusion problem leads to these extreme solutions.

Therefore, we consider also the minimization of an associated regularized objective function

$R_{\text {reg }}=\int_{T} \int_{\mathrm{CMB}}\left(B_{r}^{\mathrm{obs}}-\hat{B}_{r}\right)^{2} \mathrm{~d} S \mathrm{~d} t+\lambda \int_{R^{3}} \hat{\mathbf{B}}_{0}^{2} \mathrm{~d} V$,

where the second term corresponds to penalizing the energy of the initial magnetic field $\hat{\mathbf{B}}_{0}$ over all space, scaled with a damping parameter $\lambda$. Note that for purely diffusive SV the total magnetic energy monotonically decays with time (e.g. Gubbins \& Roberts 1987) and this regularization term will therefore penalize magnetic energy over the entire time period. Nevertheless, this constraint still permits any local exchange of magnetic energy, such as the overall energetic growth at the CMB observed for the 20th century (Huguet et al. 2018). The choice of this regularization differs from that adopted elsewhere, for example, the dissipation norm of Gubbins \& Bloxham (1985) and is motivated here by the very simple structure (see Appendix D for a derivation):

$\int_{R^{3}} \hat{\mathbf{B}}_{0}^{2}=\hat{\mathbf{q}}^{T} \hat{\mathbf{q}}$

The discretized regularized objective function is then

$R_{\mathrm{reg}}=(\mathbf{g}-\mathbf{D} \hat{\mathbf{q}})^{T} \mathbf{W}(\mathbf{g}-\mathbf{D} \hat{\mathbf{q}})+\lambda \hat{\mathbf{q}}^{T} \hat{\mathbf{q}}$,

whose minimum with respect to $\hat{\mathbf{q}}$ then yields

$\hat{\mathbf{q}}=\left(\mathbf{D}^{T} \mathbf{W D}+\lambda \mathbf{I}_{N L(L+2)}\right)^{-1} \mathbf{D}^{T} \mathbf{W g}$,

where $\mathbf{I}_{p}$ is the $p \times p$ identity matrix, with $p=N L(L+2)$. 


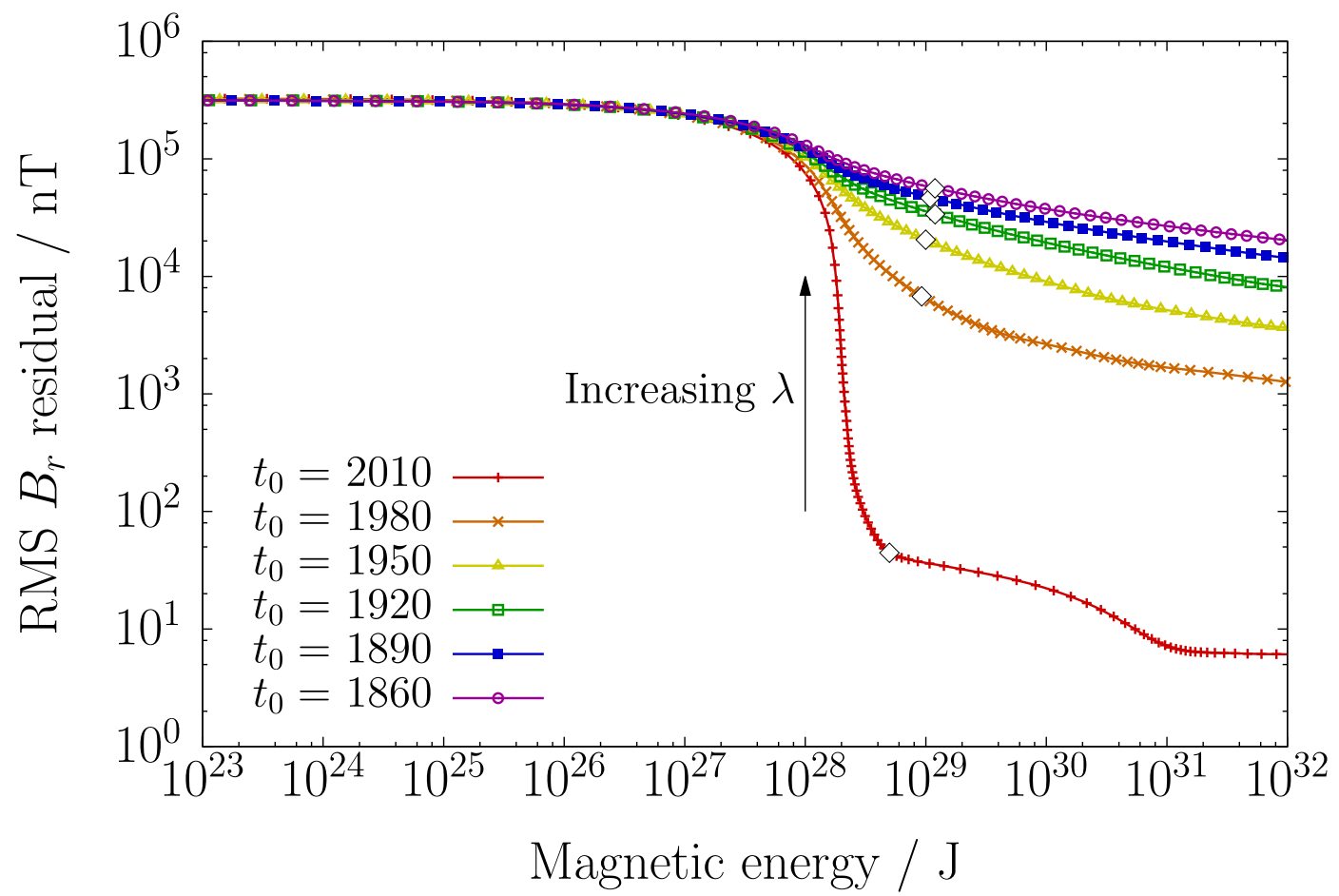

Figure 5. The RMS residual of $B_{\mathrm{r}}$ as a function of the initial magnetic energy of the solution for several time windows (from $t_{0}$ to 2015.0). Every datum represents a choice for the damping parameter $\lambda$. The diamonds indicate our preferred trade-off between the residual and regularization term.

\subsubsection{Choosing the damping parameter}

The solutions (eq. 22) parametrize a 1-D family of solutions that minimize the associated objective function $R_{\text {reg. }}$. We now address which solution we should choose. Ultimately, we are interested only in the solution that best describes SV with realistic amplitudes in the modelled core magnetic structure. In the following, we shall find values for this parameter we consider optimal based on how $\lambda$ affects the trade-off between the residual and the regularization term.

Fig. 5 shows a trade-off curve for $\lambda$ showing the RMS residual in $B_{\mathrm{r}}$ [i.e. the square root of $R_{\text {unreg }}$ (eq. 16) averaged over the CMB and $T]$ as a function of the associated initial magnetic energy (defined over all space) for various time windows, where every datum represents a choice of $\lambda$. These models have been calculated using double (16-digit) precision, 30 basis functions and 201 time points (these values ensure a numerically converged solution, see Appendix C). We note that for the data plotted, the regularization term ensures that double-precision is sufficient: clearly as $\lambda \rightarrow 0$ (and in fact for values of the energy in excess of roughly $10^{32} \mathrm{~J}$ ) we recover approximately the unregularized problem for which much higher precision is needed. For all time windows, there appears to exist a regime in which the solutions do not contain enough energy to allow an adequate fit to the observations, typically when the energy is below $10^{27} \mathrm{~J}$. Decreasing $\lambda$ and so increasing the energy beyond this point to roughly $10^{29} \mathrm{~J}$, yields a significant reduction in the residual. This is particularly notable for short time windows; allowing increased energy in the longer time windows results in a relatively low improvement in the residual.

For all time windows, we consider $\lambda$ optimal if it is on the 'knee' of the trade-off curve after the initially rapid decline of the residual (diamonds in Fig. 5). This knee is difficult to define quantitatively for time windows spanning several decades; therefore, all preferred values for $\lambda$ have been picked by eye, which range between $10^{-18}$ and $10^{-3}$ (Supporting Information data file 1 lists these values). Lastly, we note that all our preferred models have a full-core RMS field strength between 0.6 and $1.3 \mathrm{mT}$ and are therefore consistent with the 2.5 or $4 \mathrm{mT}$ estimates provided by Gillet et al. (2010) and Buffett (2010).

\section{OPTIMIZED PURELY DIFFUSIVE MODELS}

\subsection{Regularized models}

Here we present a set of purely diffusive models comprising regularized inversions for an initial magnetic structure whose subsequent evolution is described by the mean COV-OBS.x1 model. These solutions have been obtained for varying time windows terminating at 2015.0 and have been computed with 30 basis functions, 201 time points (ensuring a numerically converged solution, Appendix C) and optimal $\lambda$ values that have been selected using the criteria presented in Section 2.4. For these models, we show a number of results. First, we compare time-series of selected Gauss coefficients over a single time window. Second, we present $B_{\mathrm{r}}$ residual error spectra for several time windows. 

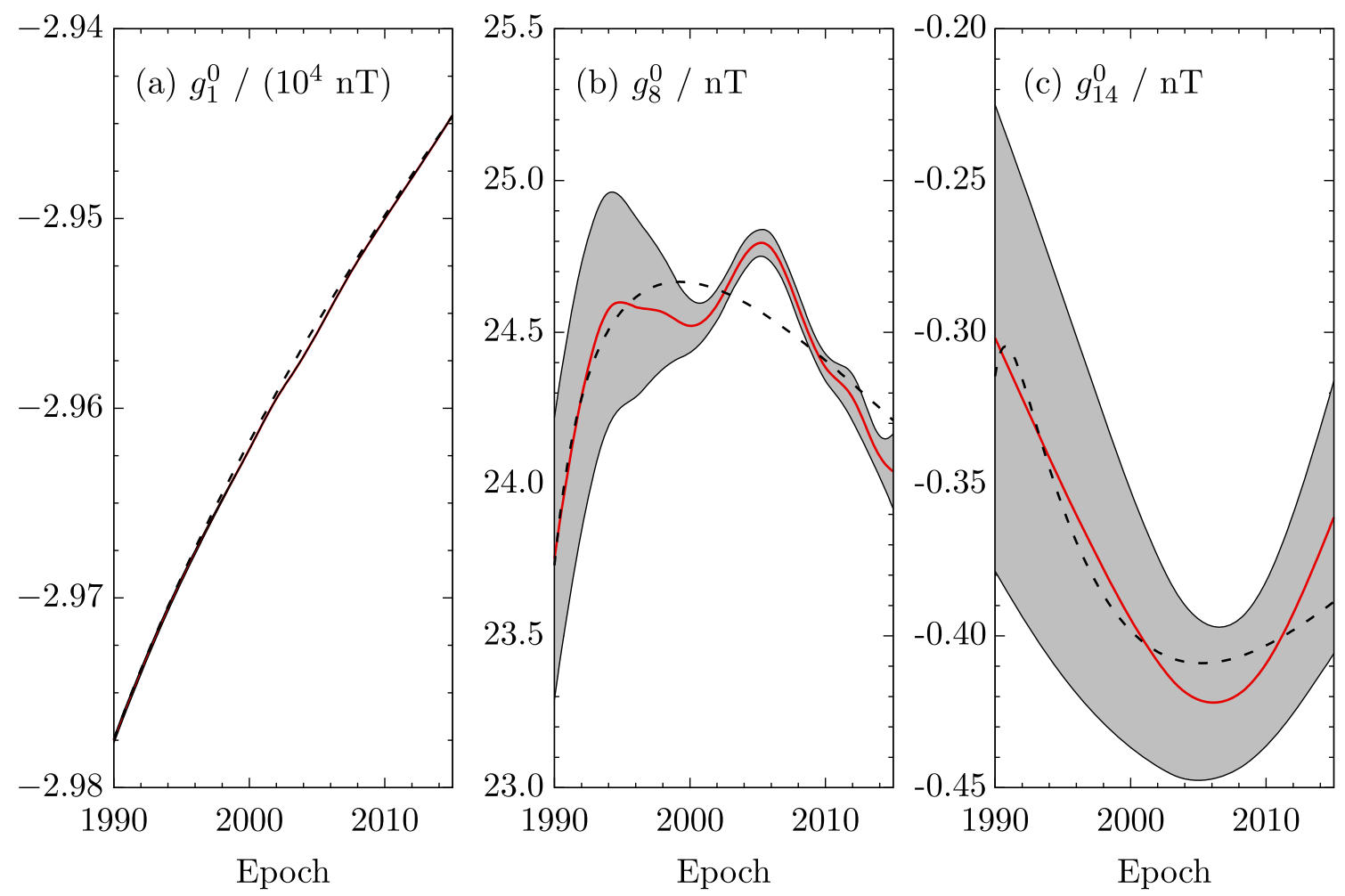

Figure 6. Comparison between time-series of selected Gauss coefficients from the regularized diffusion model for 1990.0-2015.0 (black dashed) and the mean COV-OBS.x1 model (solid red). Grey shaded areas represent the mean COV-OBS.x1 model \pm one standard deviation.

We also demonstrate how the total residual changes as a function of the window length. Finally, we map $B_{\mathrm{r}}$ and $\dot{B}_{\mathrm{r}}$ residuals at the CMB and at selected epochs.

Fig. 6 shows time-series of the Gauss coefficients $g_{1}^{0}, g_{8}^{0}$ and $g_{14}^{0}$, for the period 1990.0-2015.0 from the COV-OBS.x1 mean (solid red) and diffusive models (dashed black). The grey shaded areas correspond to the time-dependent one standard deviation error bounds. Despite the correct qualitative behaviour of the diffusive model, only the high-degree coefficients are fit within the COV-OBS.x1 uncertainty. For instance, while the regularized diffusion model captures the overall decay of $g_{1}^{0}$, the diffusive time-series is generally outside the COV-OBS.x 1 error budget. Conversely, the regularized fit to $g_{14}^{0}$ is well within the COV-OBS.x1 error budget at all times. This effect is in part driven by the relative increase in the COV-OBS.x 1 error budget with degree: for degree 1 the relative uncertainty is small, typically $<0.1$ per cent for this period, whereas for degree 14 it may be as large as 25 per cent. Nevertheless, the diffusive fits to $g_{1}^{0}$ and $g_{8}^{0}$ also lack the short-term variability required to fit COV-OBS.x1 within the uncertainty at all times.

That diffusion captures higher degree features of the field more easily is quantified with spherical harmonic error spectra (Fig. 7), which have been computed for various time windows using

$E\left(l_{\alpha}\right)=\frac{\left(l_{\alpha}+1\right)^{2}}{2 l_{\alpha}+1}\left(\frac{a}{c}\right)^{2 l_{\alpha}+4} \sum_{m_{\alpha}=0}^{l_{\alpha}} \frac{1}{T} \int_{T}\left(g_{\alpha}(t)-\hat{g}_{\alpha}(t)\right)^{2} \mathrm{~d} t$.

This spectrum relates to the residual in eq. (16): the sum of $E\left(l_{\alpha}\right)$ over $l_{\alpha}$ is the mean squared error in $B_{\mathrm{r}}$ averaged over $T$ and the CMB. Shown as the solid curves in Fig. 7 are also the COV-OBS.x1 uncertainty spectra computed with eq. (23) and with the integrand taken to be the variance; the total mean squared uncertainty is then the sum of the uncertainty spectrum. Readily notable is the contrast of rather flat diffusion error spectra, and the COV-OBS.x1 uncertainty spectra increasing monotonically with $l_{\alpha}$, demonstrating that high-degree features are more likely to be fit within the uncertainty. Also, for all degrees, the average error increases when a longer time window is considered, therefore for longer periods only relatively high-degree features are captured well: for 2010.0-2015.0 degrees 3 to 14 are within the uncertainty bounds; for 1985.0-2015.0 only degrees 10 to 14 are within these bounds.

While for most degrees the regularized 1985.0-2015.0 error spectrum is orders of magnitude larger than the COV-OBS.x1 uncertainty, the total residual is actually smaller than the total uncertainty based on a single standard deviation. We confirm this by showing for the complete set of diffusion models the time-averaged RMS residual as a function of time window length, together with this uncertainty (Fig. 8). It then becomes evident that with our regularized diffusion models, we may fit SV over $102 \mathrm{yr}$, without producing a time-averaged residual greater than the COV-OBS.x1 error budget based on one standard deviation. Additionally, we find 30 yr to be the longest time window for which regularized diffusion fits SV while the total residual is smaller than the total uncertainty at all points in time. Nevertheless, it is of note that if we consider the 1913.0-2015.0 (102 yr) diffusive model and compute the associated time-averaged residual over a shorter period 


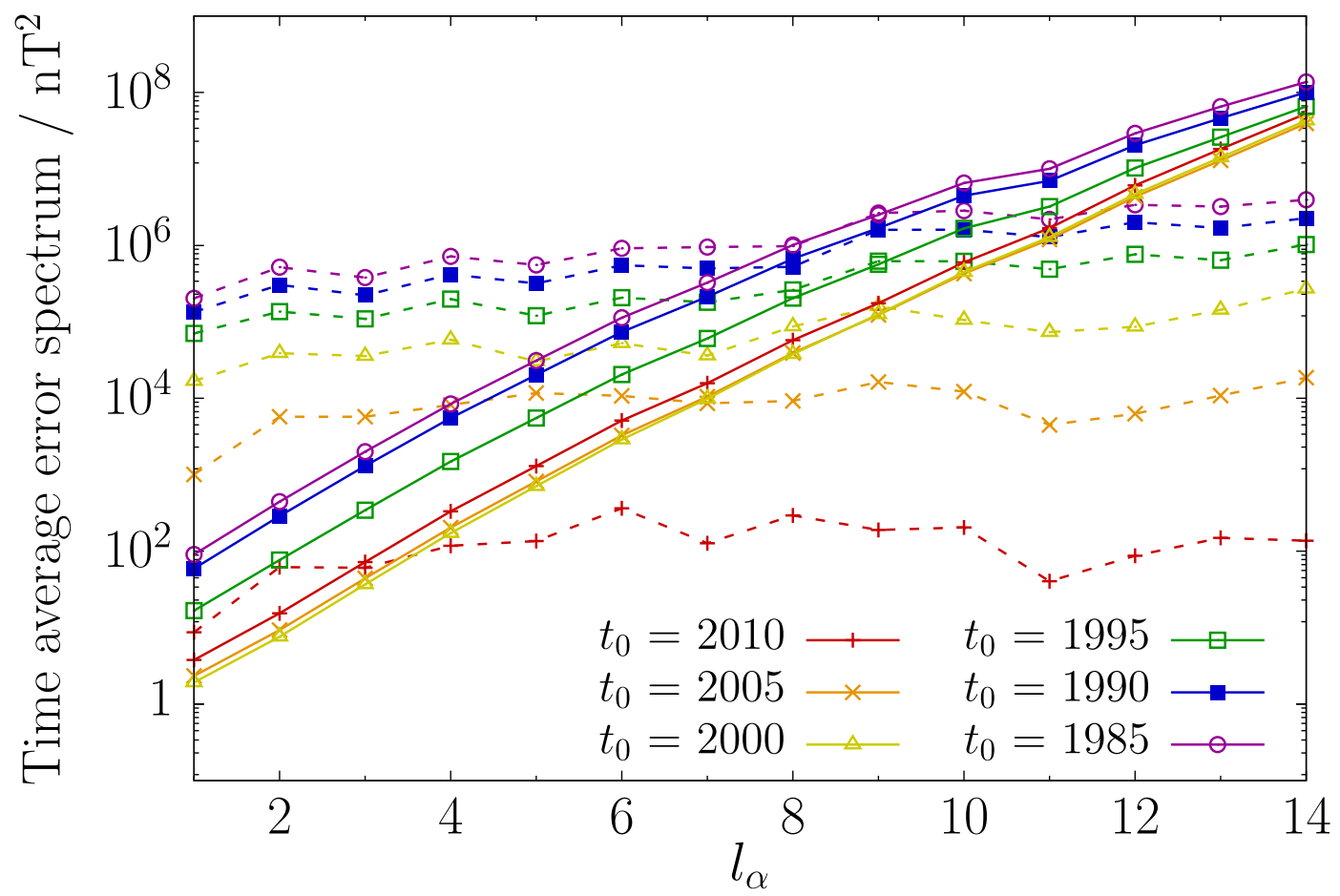

Figure 7. Time-averaged spectra of the residual $B_{\mathrm{r}}$ at the CMB for the regularized diffusion models (dashed) and the time-dependent standard deviation of COV-OBS.x1 (solid). The line colours and symbols represent different time windows spanning from $t_{0}$ to 2015.0.

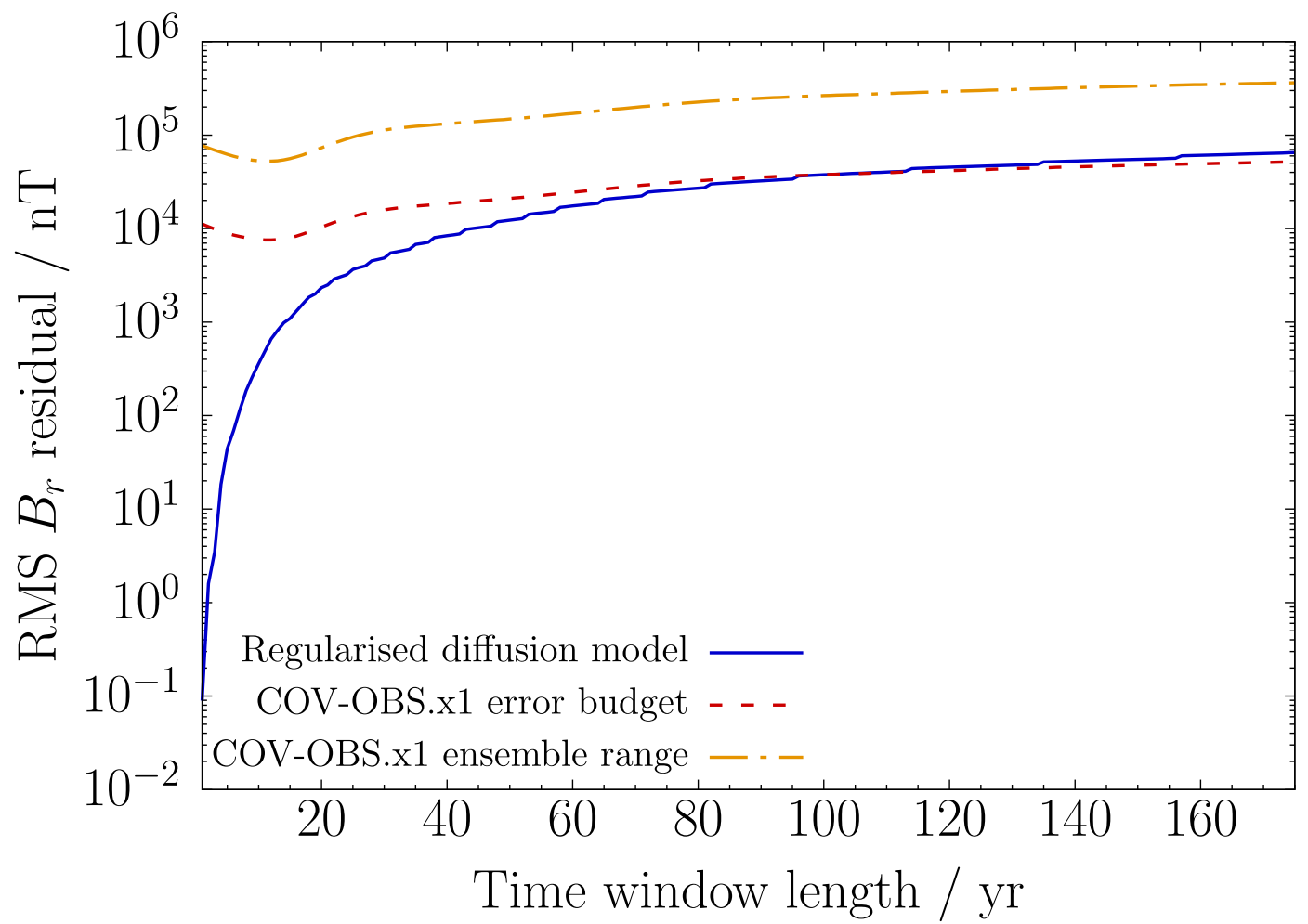

Figure 8. The time average of the RMS $B_{\mathrm{r}}$ residual over the CMB as a function of time window length for the regularized diffusion models (solid blue). Also shown are two time-average global uncertainties for COV-OBS.x1, one computed with one standard deviation among Gauss coefficients (dashed red) and the other from maximum differences between the 100 published ensemble members and the ensemble mean (dot-dashed yellow). 
(here taken as 1990.0-2015.0), we find it amounts to $4.78 \times 10^{4} \mathrm{nT}$ - now roughly 2.5 times as large as the total COV-OBS.x1 uncertainty for the 1990.0-2015.0 period. A similar procedure but with these residuals evaluated at the Earth's surface yields an average of 810.5 nT for the full period; when averaged over the 1990.0-2015.0 period with the use of the same model, this residual equals approximately $1162 \mathrm{nT}$. These differences are indicative of an overall temporal growth of the residual, which we find for almost any choice of $T$. Shown in Fig. 8 is also a more generous global uncertainty measure computed using eq. (23) based on the maximum difference between the 100 published COV-OBS.x1 ensemble members and the ensemble mean. It can be readily seen that the regularized diffusion models fit COV-OBS.x1 within this uncertainty bound over the full 1840.0-2015.0 period.

As the total error and its associated spectrum describe how well diffusion fits only in an average sense, we illustrate also how the residual varies spatiotemporarily. In Fig. 9 the radial field is shown at the CMB and at selected epochs, as prescribed by COV-OBS.x1 and the regularized 1990.0-2015.0 model (a,d,g and b,e,h respectively; see also animation S1, Supporting Information). Also shown at these epochs is the corresponding unsigned difference between these two models (Fig. 9c, f and i; see also animation S2, Supporting Information). We find good global agreement between the models through visual comparison; this is also reflected by the associated error amplitudes, which are low compared to the radial field itself. Still, we observe an overall growth of the unsigned residuals with time, these errors being the largest towards the end of the period. Spatially, the residuals describe a pattern where the largest amplitudes reside in the Indo-Atlantic Hemisphere, in particular under Siberia, and the Pacific generally accommodating lower amplitudes. These high error amplitudes beneath the southern Indian Ocean and Asia correlate with regions for which significant secular acceleration has been reported (Olsen \& Mandea 2008). As such, Fig. 9 demonstrates that it is more difficult for the diffusion model to replicate COV-OBS.x1 where it locally describes relatively fast field change.

We confirm this statement by investigating the fit of SV on the CMB. Fig. 10 shows $\dot{B}_{\mathrm{r}}$ on the CMB and at selected epochs for COVOBS.x1 and the regularized 1990.0-2015.0 diffusion model (a, d, g, and b, e, h, respectively; see also animation S3, Supporting Information); the associated unsigned SV differences are given in c, f and i (see also animation S4, Supporting Information). The residual patterns in Fig. 9 bear resemblance to the SV described by COV-OBS.x1 as both are characterized by relatively large amplitudes under Siberia, the South Atlantic and the Indian Ocean, in particular at 2015.0. Furthermore, the residual SV amplitudes are relatively large compared to $\dot{B}_{\mathrm{r}}$, although it is of note that we do not explicitly minimize the SV residual. Particularly striking is the initially high amplitude SV described by the diffusion model, which is associated with large residuals at 1990.0. However, these amplitudes diminish quickly, and we find reasonable global agreement midway through the time period. The diffusion models tend to produce higher SV initially, which diminishes through time, such that they best explain the rate of change near the centre of the modelling period. The SV residuals increase in magnitude towards the end of the period, and by 2015.0 it is particularly difficult for the diffusion model to match the SV amplitudes beneath Siberia.

\subsection{Comparison with core field characteristics and evolution}

The results presented above, and in particular Fig. 8, suggest that a globally optimized field structure with an associated diffusive time dependence is consistent with core field evolution on yearly to decadal timescales. In this section, we focus on how certain local features of the field are represented by these diffusive models.

\subsubsection{Depth extent of reversed flux patches}

Fig. 11 shows the initial $\left(t=t_{0}\right)$ radial field for the regularized 1990.0-2015.0 diffusive model at selected depths inside the core (see also animation S5, Supporting Information). Because this model is expected to be poorly resolved below roughly $3400 \mathrm{~km}$ radius (see Appendix A), we only highlight the modelled field structure between this depth and the CMB. In general, the field is found to contain more structure and to be of higher amplitude at greater depth. Additionally, the axial dipole is less prevalent within deeper regions of the core, as reversed-flux patches (RFPs; Gubbins 1987; Olson \& Amit 2006; Terra-Nova et al. 2015; Metman et al. 2018), that is, areas horizontally bounded by null-flux curves where the sign of $B_{\mathrm{r}}$ is opposite to the one expected from the dipole component of the field show a marked expansion and proliferation with increasing depth. We find in our regularized inversions that some of the RFPs on the CMB extend down to the limit of our resolving depth of $85 \mathrm{~km}$ inside the core; indeed, patches can merge or separate as depth increases.

\subsubsection{Westward drift}

One of the most characteristic features of historical field evolution is the secular drift of the field towards the west, prevalent particularly within the Atlantic equatorial region. This overall westward movement of the low-latitude field is also manifest in COV-OBS.x 1 as may readily be observed by evaluating the associated radial field at the equator as a function of time and longitude (Figs 12a and c). The regularized 1913.0-2015.0 diffusion model, which fits COV-OBS.x1 within its uncertainty based on the global time-averaged metric, is able to locally reproduce the $0.2^{\circ}$ drift rate (Fig. 12b). However, this model only provides realistic drift rates during approximately the first 40 yr of the modelling period, after which the model's diffusive azimuthal field motion decelerates. Nevertheless, the total westward displacement of the low-longitude field is matched reasonably. If we consider a shorter time window (e.g. 1990.0-2015.0), the corresponding regularized diffusion model gives the correct drift rates at all times (Fig. 12d). 

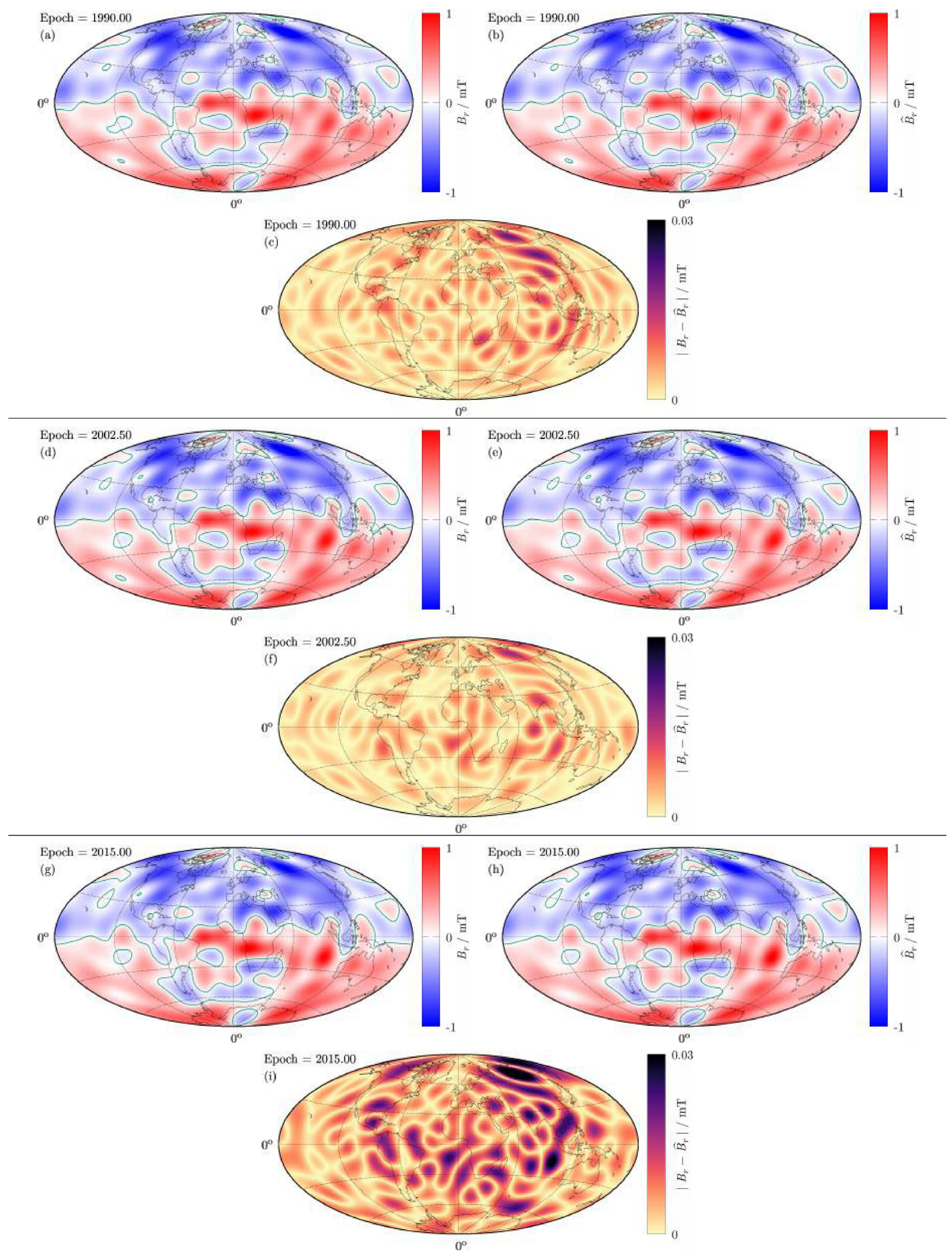

Figure 9. The radial field at the CMB and at selected epochs as described by COV-OBS.x1 (a,d,g) and the regularized 1990.0-2015.0 diffusion model (b,e,h); figures c, f and i show the corresponding unsigned difference between the two models. Null-flux curves are represented in green. 

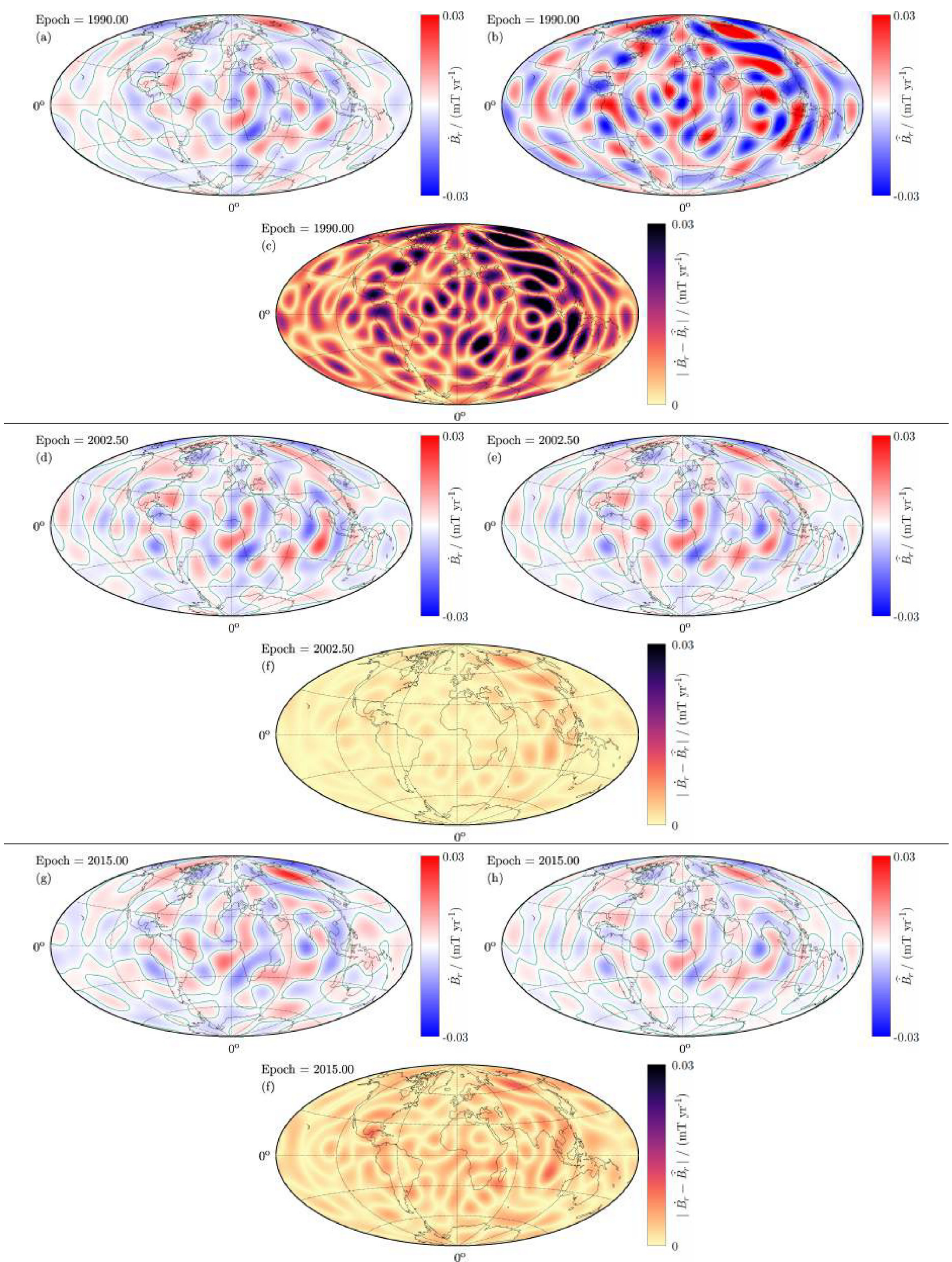

Figure 10. As Fig. 9, but for the time derivative of the radial field. 

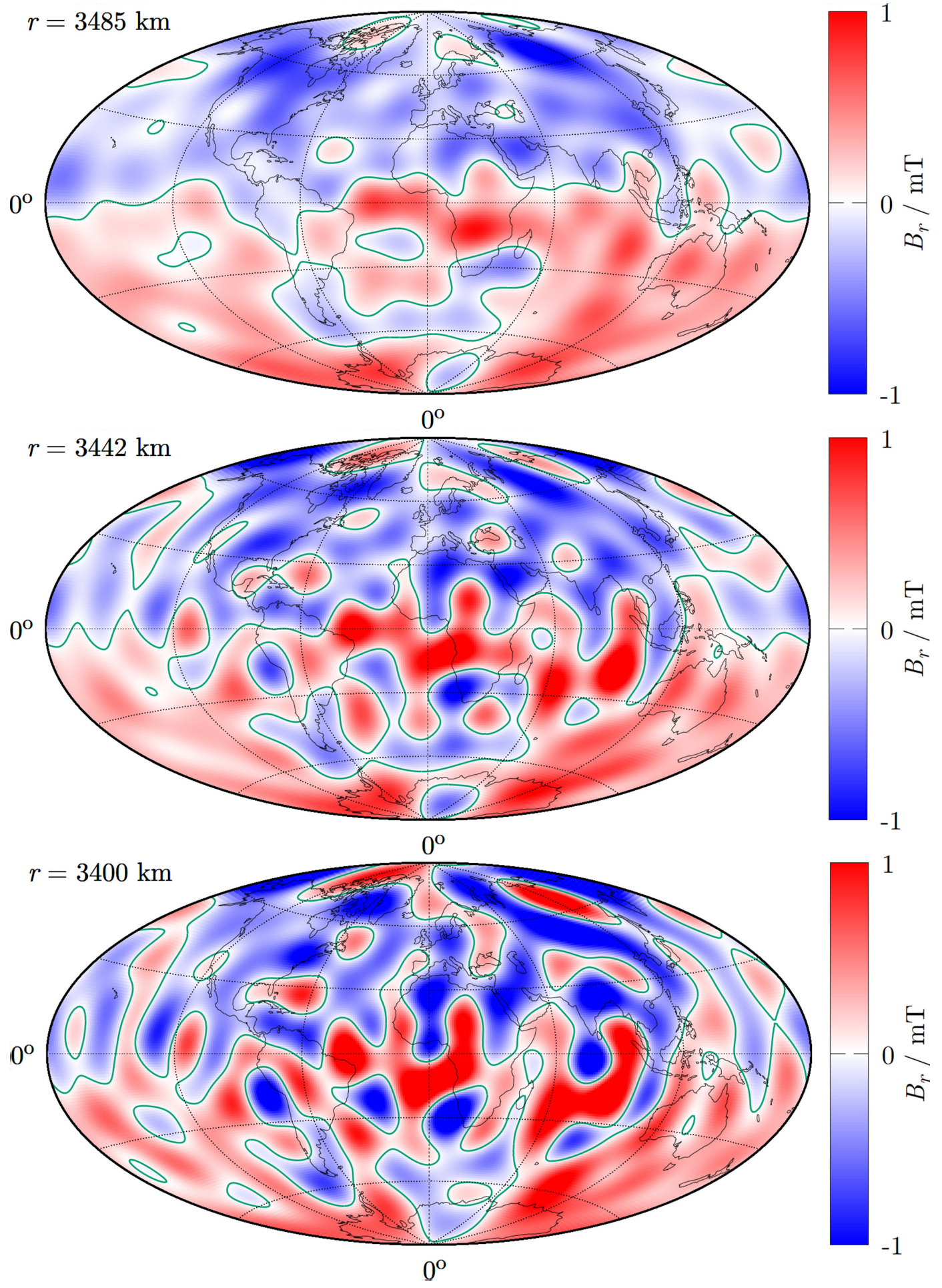

Figure 11. The radial field $B_{\mathrm{r}}$ from the regularized 1990.0-2015.0 diffusion model at $t=t_{0}$ and selected depths inside the core. Null-flux curves are represented in green.

\subsubsection{Recent North Magnetic Pole acceleration and Arctic emergence of reversed flux}

The NMP, that is, the location on Earth's surface where the magnetic field vector points vertically downwards, has been shown to have accelerated over the past three decades (e.g. Mandea \& Dormy 2003). The 1913.0-2015.0 regularized diffusion model appears incompatible with this trend, as the NMP moves relatively slowly for this model, and ultimately stalls almost entirely (Fig. 13a, contoured circles). However, if we focus on the period during which pole acceleration occurred, for example, for the period 1990.0-2015.0, the regularized diffusion model shows an NMP trajectory with much improved agreement with COV-OBS.x1 (Fig. 13b). However, there remains some discrepancy between 

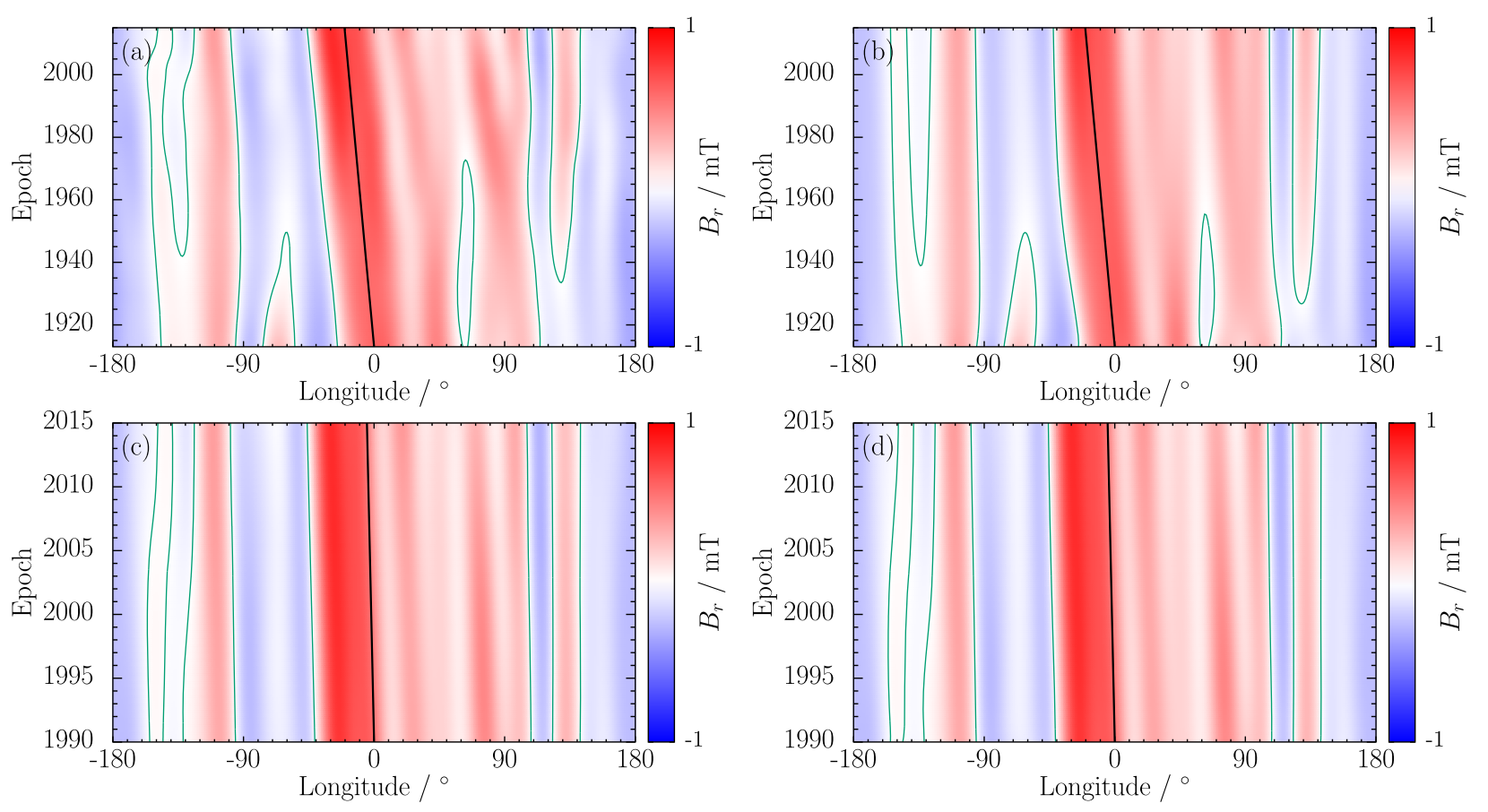

Figure 12. Time-longitude plots of the radial field at the equator according to COV-OBS.x1 (a and c) and the regularized 1913.0-2015.0 and 1990.0-2015.0 diffusion models ( $\mathrm{b}$ and d, respectively). The black line corresponds to a drift rate of $0.2^{\circ} \mathrm{yr}^{-1}$ towards the west. Null-flux longitudes are represented in green.
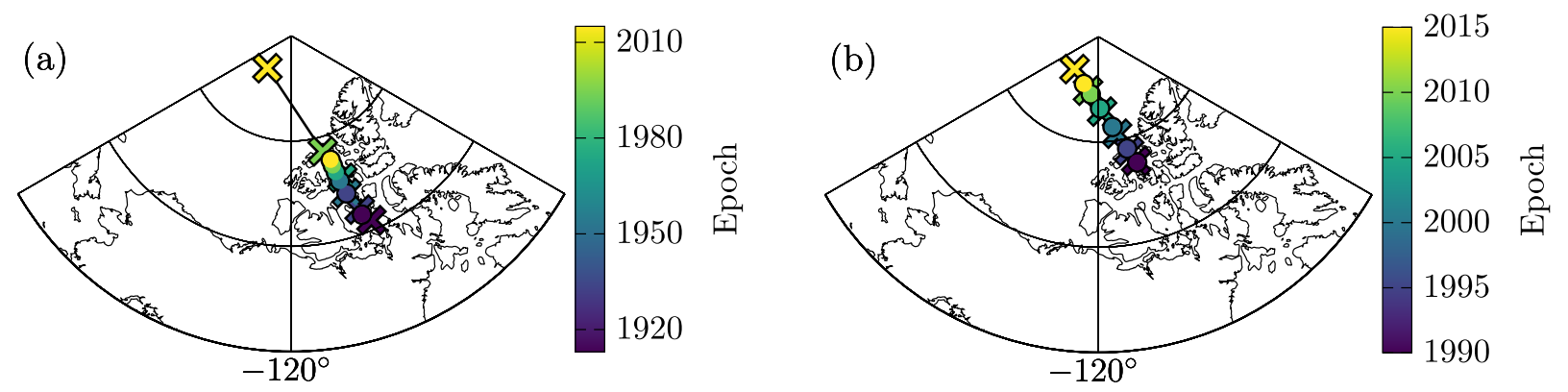

Figure 13. The position of the North Magnetic Pole on the Earth's surface as a function of time, according to COV-OBS.x1 (crosses; a and b) and the regularized diffusion models for 1913.0-2015.0 and 1990.0-2015.0 (circles; a and b, respectively). The colour of the crosses and circles denotes time.

the two models at the end of the period, with the NMP from the diffusive model decelerating and unable to match the COV-OBS.x1 pole location at 2015.0.

Chulliat et al. (2010) attribute the NMP acceleration to growth and intensification of a patch of reversed flux under the New Siberian Islands. The recent evolution of this patch is shown in Fig. 14, an equal area projection of $B_{\mathrm{r}}$ at selected epochs according COV-OBS.x1 (a, $\mathrm{c}$ and e) and the 1990.0-2015.0 diffusion model (b, d and f). Both field models highlight in almost identical fashion how the New Siberian Islands patch expands and intensifies. Additionally, for both models we observe a net migration of this patch towards the southwest. For the diffusion model, the evolution of the patch can be linked to field morphology at greater depth (Fig. 11): it should be noted that the patch extends to our sensitivity depth limit of $85 \mathrm{~km}$, where it is of higher amplitude and displaced towards the southwest compared to the field configuration at the $\mathrm{CMB}$, so that subsequent diffusive evolution of this initial state will yield the intensification and migration displayed in Fig. 14.

\subsubsection{Surface geomagnetic time-series and the late 20th century jerk}

Finally, we examine how well a globally optimized diffusion model may capture the local time-series of a single field component at the Earth's surface. Specifically, we consider once more the regularized 1990.0-2015.0 diffusion model and the COV-OBS.x1 mean, and its representation of the eastward field component $Y$ at the Chambon La Forêt (CLF) observatory (Fig. 15a). Our interest is focused on a geomagnetic jerk, that is, a stepwise change in the second time derivative of a magnetic field component, which has been observed at this station at approximately 1999.0 (Mandea et al. 2000). We therefore compare also the time derivative $\dot{Y}$ (Fig. 15b). The diffusive model captures the magnitude and growth of $Y$ in a general sense, although the two curves clearly diverge towards the end of the time period. However, the time derivatives 

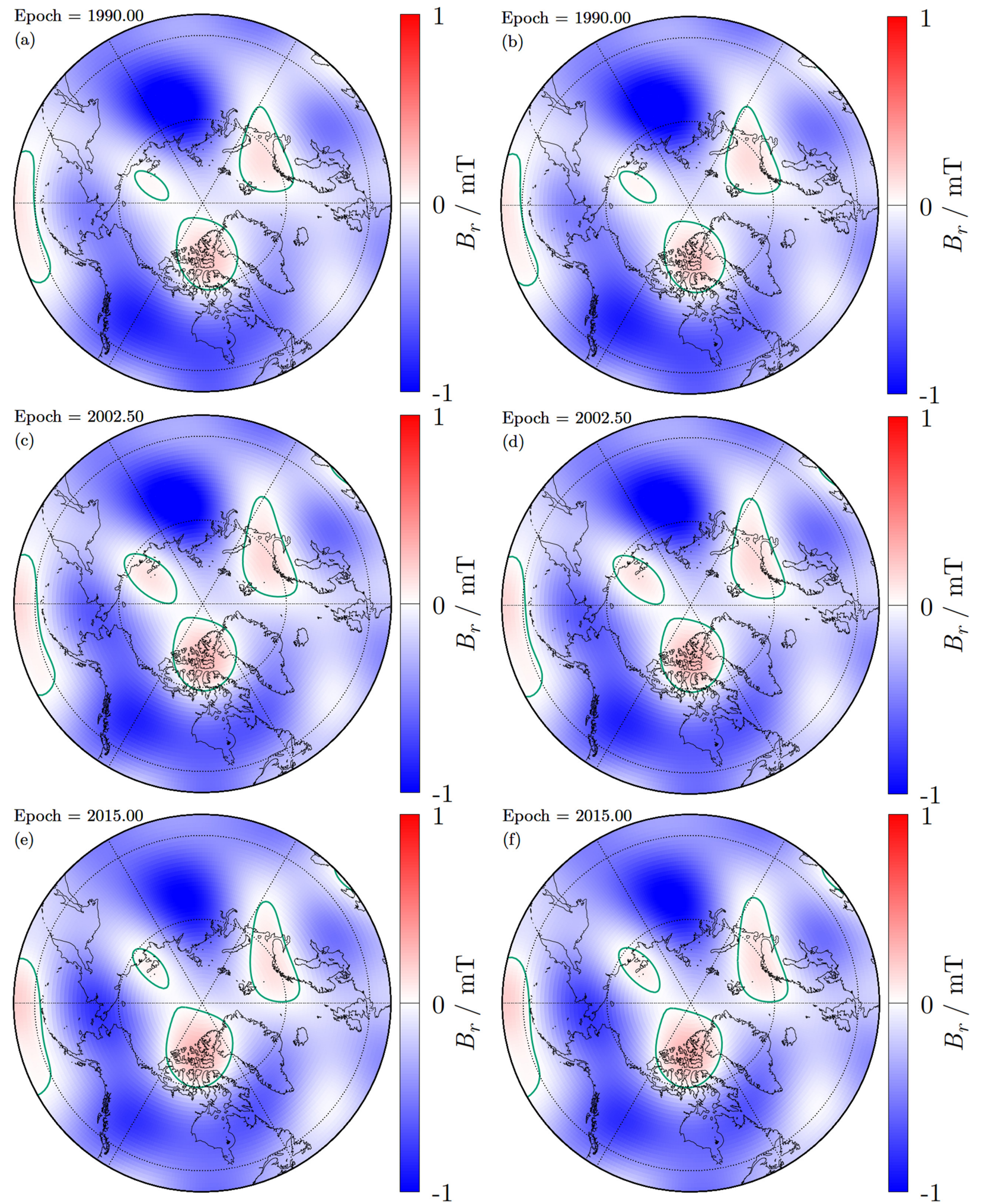

Figure 14. Polar projection of $B_{\mathrm{r}}$ at the CMB and selected epochs for COV-OBS.x1 (a, c and e) and the 1990.0-2015.0 diffusion model (b, d and f). Null-flux curves are represented in green.

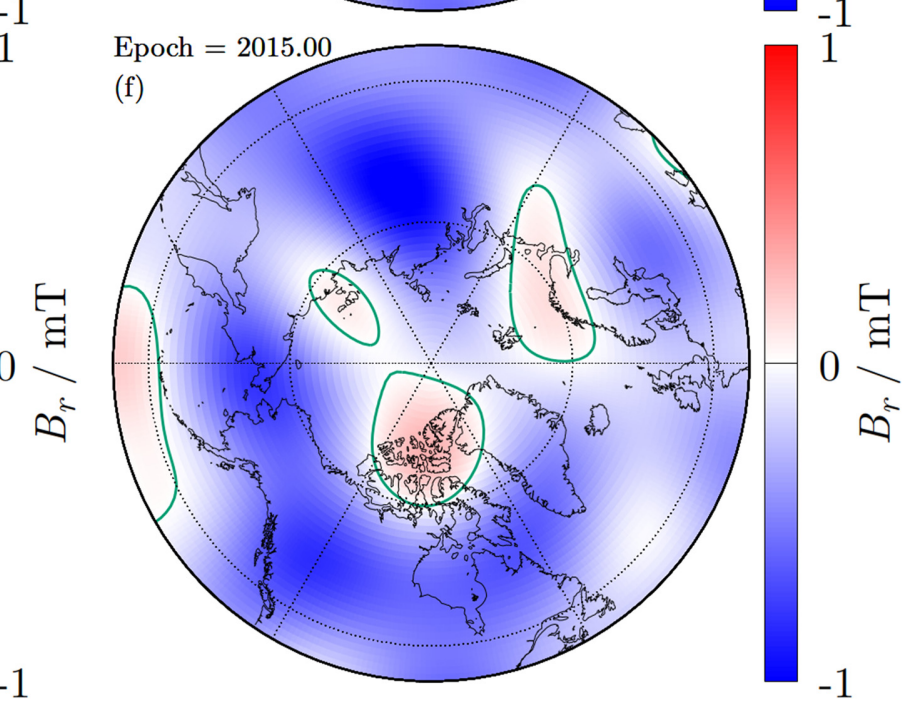

Epo

(d)

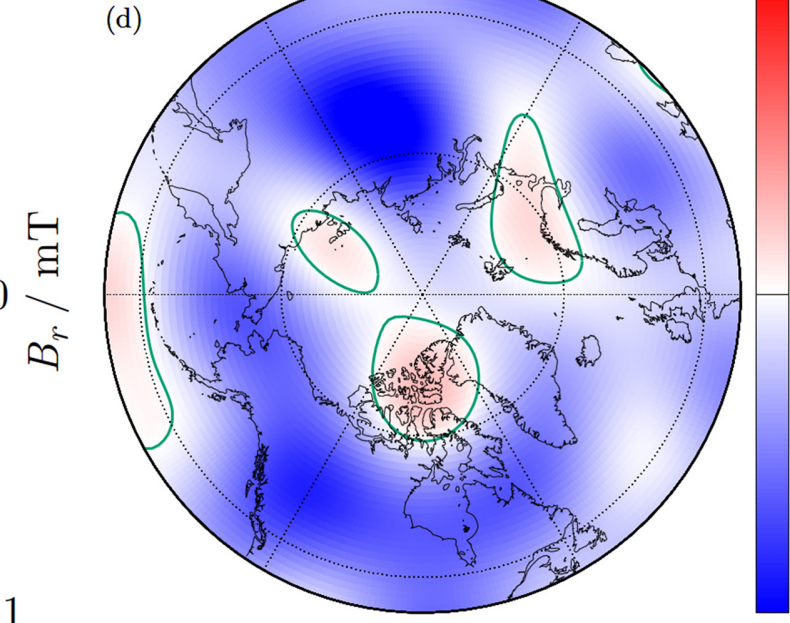

0

$\operatorname{li}^{2}$

1

Epoch $=2015.00$ 

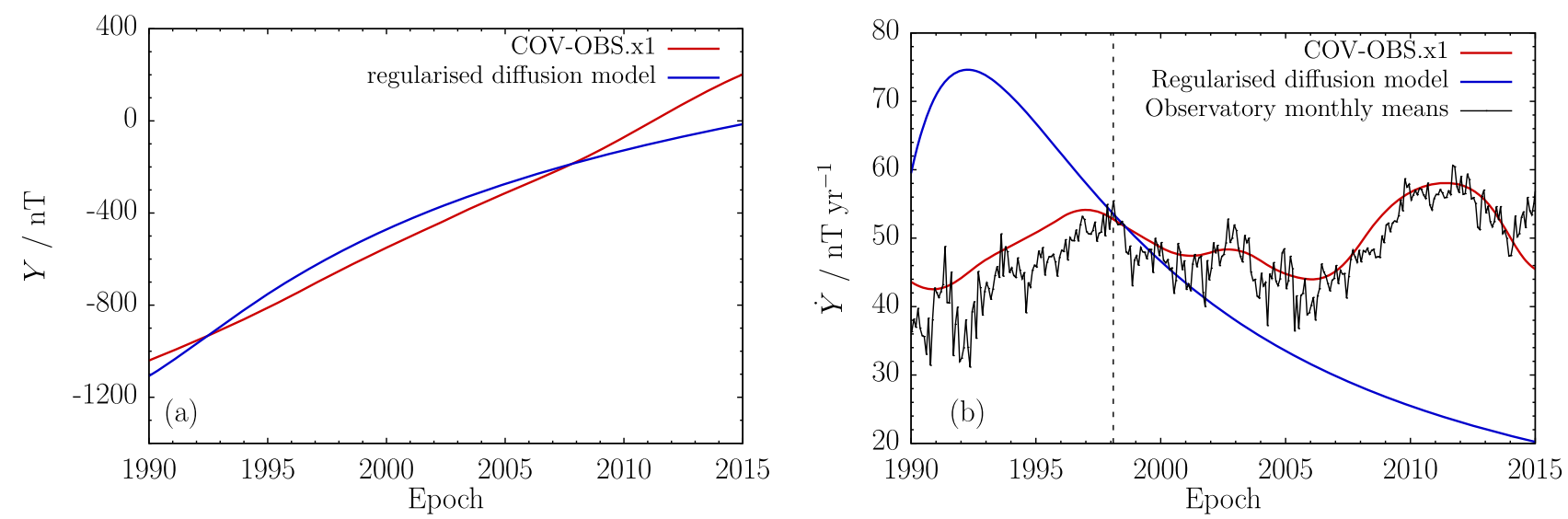

Figure 15. Time-series of the eastward magnetic component $Y$ (a) and its time derivative (b) at the Chambon La Forêt (CLF) observatory, according to COV-OBS.x1 (red), the regularized 1990.0-2015.0 diffusion model (blue) and first differences of observatory monthly means (black). The dashed line denotes the approximate timing of a geomagnetic jerk.

for both models show little agreement; COV-OBS.x1 has more short-term variability with no significant overall trend, whereas the diffusive model has generally smoother behaviour and an overall decay. Although the diffusive model renders an abrupt change in $\dot{Y}$ during the first $2 \mathrm{yr}$, it is too early and of significantly different magnitude to resemble the late 20 th century jerk.

\section{DISCUSSION}

We have introduced new, purely diffusive, end-member models for geomagnetic SV, which are obtained by minimizing a global residual objective function with respect to the magnetic field structure at the start of the given time window. For chosen time periods up to $102 \mathrm{yr}$, these states are found to be globally consistent with the COV-OBS.x1 field model, as their associated time-averaged residual is smaller than the time-averaged COV-OBS.x1 uncertainty based on a single standard deviation. Additionally, for a $30 \mathrm{yr}$ time window, we find the global diffusive residual to be within the COV-OBS.x1 uncertainty at all times. Although these diffusive models are designed to fit COV-OBS.x1 on a global scale, they exhibit consistency with regional-scale field evolution. For instance, diffusion appears capable of reproducing much of the secular westward drift of the equatorial field over the 1913.0-2015.0 period. As such, it demonstrates that westward drift cannot be uniquely attributed to fluid flow along the CMB, although this is the most common interpretation (Bullard et al. 1950; Jault et al. 1988; Aubert et al. 2013). Moreover, our diffusive models recover both the recent shift of the NMP and the strengthening of reversed flux in the Arctic region, in agreement with the idea that these two phenomena may be linked dynamically (Chulliat et al. 2010).

However, we also considered the local fit of our models at a single location on the Earth's surface. In particular, we showed that our models could not reproduce the signature of a late 20th century jerk. While jerks remain poorly understood, we consider it unlikely that these are generated through regional-scale magnetic diffusion. However, it should be noted that within our formalism, observatory time-series are not fit directly; indeed, it may be possible to fit a local time-series of SV at the expense of large global residuals. Another issue is that we minimize a global residual with respect to the field model COV-OBS.x 1 that is already a spatiotemporarily smoothed representation of the true core field and its time evolution. The models presented here are therefore the result of an indirect inversion and have accordingly undergone additional (implicit) regularization. This secondary smoothing may explain how the models fit well globally but still produce a large misfit to field evolution at one particular location. A potential modification of our formalism is to directly minimize the misfit to ground and/or virtual satellite observatory (Mandea \& Olsen 2006) data instead of an intermediate spherical harmonic field model, which may confirm with more confidence how well magnetic diffusion alone can fit local geomagnetic time-series.

Let us briefly compare how well field evolution is described by both SV end members, which relate to either frozen flux or magnetic diffusion. Whaler \& Beggan (2015) and Beggan \& Whaler (2018) report frozen-flux fluid flow and acceleration models with associated RMS hindcast errors in $|\mathbf{B}|$ at the Earth's surface on the order of tens of nT, over a period of 5 yr. Evaluating our 5-yr (2010.0-2015.0) regularized diffusion model at the surface, we find the respective diffusive error is only $1.3 \mathrm{nT}$. It is important to recognize however that our diffusive models have more degrees of freedom compared to these particular frozen-flux models. Choosing a coarse radial resolution for the diffusive models $(N=2)$ in order that the number of degrees of freedom are the same produces a 5-yr time-averaged RMS error in $|\mathbf{B}|$ at the Earth's surface of almost 93 nT. However, there is no physical reason why we should deliberately penalize our diffusive models in such a comparison. Furthermore, the simple measure of global misfit does not take into account how well either method captures the evolution of local features: either method could be superior in describing SV within a specific geographic area. Assuming the models by Whaler \& Beggan 
(2015); Beggan \& Whaler (2018) are illustrative of the broader suite of core flow models that have been developed (see Holme (2015) for an overview), we conclude that the diffusive end-member model of SV provides an equally acceptable mathematical description of decadal field evolution. Additionally, our results confirm that diffusion need not be restricted to the global timescale of $\tau_{\mathrm{d}} \approx 16 \mathrm{kyr}$. Furthermore, the effective $R m$ near the top of the core could be much lower than canonical estimates, because fine radial magnetic field structure reduces the typical length scale and when diffusion explains a significant amount of the SV large-magnitude flows are not required. Hence, for our purely diffusive models $R m$ is much closer to estimates of a local $R m \sim 1$ that apply just below the CMB (e.g. Amit \& Christensen 2008; Finlay \& Amit 2011).

Although both frozen-flux and purely diffusive models may adequately describe field evolution, neither end member of SV should be considered fully representative of the physical processes that govern the geodynamo. For example, while fluid flow can (re)generate the magnetic field, diffusion enters only as a sink term in the description of total magnetic energy evolution (e.g. Gubbins \& Roberts 1987). Nevertheless, in the absence of fluid flow there may still be a local energetic exchange, as demonstrated by the regularized 1840.0-2015.0 diffusion model, for which we find an overall threefold increase of the magnetic energy on the CMB. This result also contrasts with a previous suggestion that time variability in the CMB magnetic energy relates only to fluid flow (Huguet et al. 2018). However, it should be noted that such local purely diffusive field growth is inherently transient, which explains the observed overall increase of modelling errors with time (e.g. in Figs 6, 9, 12 and 15a), and the increased difficulty of using diffusion to explain field evolution over relatively long time windows (Fig. 8).

The dissipative nature of diffusion may also be illustrated with a comparison of field states, for example, the regularized 2000.0-2015.0 model evaluated at 2010.0 and the initial state for the regularized 2010.0-2015.0 model: over the sensitivity region we find that the overall relative difference in $\mathbf{B}$ between these states amounts to roughly 72 per cent, and this large temporal discontinuity between field states may suggest that one decade is sufficient to dissipate a significant part of the initial state. As such, on longer (geological) timescales diffusion alone could not have sustained the field throughout the core's lifetime, estimated to be on the order of billions of years (e.g. Tarduno et al. 2010; Biggin et al. 2015). The evolution of the field over these geological timescales highlights a further limitation of pure diffusion, namely its inability to explain the numerous global polarity reversals that have occurred over the field's lifespan (e.g. Glatzmaier \& Coe 2015). We therefore underline once more that pure magnetic diffusion is not a self-sustainable process physically capable of explaining SV indefinitely. Furthermore, as diffusion is entirely unrelated to core fluid motion, it cannot explain the observed decadal length-of-day variations due to angular momentum exchange between the core and mantle, while the frozen-flux hypothesis has allowed these to be linked to SV (Jault et al. 1988; Jackson et al. 1993; Gillet et al. 2010). Likewise, frozen-flux does not capture magnetic field evolution completely and without diffusion it would allow small-scale features of the field to grow indefinitely, leading to its failure to match the observed field. Nevertheless, while neither SV end member can explain field evolution completely, both descriptions may be utilized to fit SV on yearly to decadal timescales.

Naturally, a more complete model would consider the combined effects of both fluid flow and diffusion that could, for example, be envisaged by considering diffusion as a correction term to frozen flux (Gubbins 1996). Alternatively, one could envisage a scheme where diffusion is not strictly corrective, but where it is instead made an arbitrary part of the SV at the CMB. Together with the methods presented here, such a partitioning of the SV would allow the frozen-flux and diffusive parts to be fit individually. By subsequently varying the amount of SV that is attributed to diffusion, a hybrid model (incorporating frozen-flux and diffusion) which best explains the total SV could be obtained.

Lastly, we comment on the relevance of our work to the proposed stratified layer at the top of the liquid core with a possible depth of several hundreds of km (e.g. Whaler 1980; Lay \& Young 1990; Gubbins 2007; Buffett 2014). Within such a layer, core fluid flow would be principally horizontal and with radial flow being suppressed, it is then not straightforward to physically justify the large radial magnetic gradients close to the CMB manifest in these initial states, which could more easily be linked to flux expulsion driven by upwelling in the case of whole-core convection. Nevertheless, our work demonstrates a mathematical consistency between diffusion and observed SV that holds in the presence or absence of such a stratified layer. The short-term evolution of the field can therefore not be used as evidence for or against a stratified layer.

To conclude, we have presented a purely diffusive description of geomagnetic SV. Our diffusive SV end-member models have been obtained through the global optimization of an initial magnetic field and have a subsequent diffusive evolution that fits the COV-OBS.x 1 field model over various time windows with a given uncertainty. While purely diffusive SV is described analytically by the decay modes, we use a Galerkin method that yields a much better conditioned inverse problem. We find that diffusion alone can adequately describe historical field evolution over time windows ranging up to $102 \mathrm{yr}$ and can reproduce characteristic aspects of SV such as westward drift, recent NMP movement and emergence of reversed-flux patches.

\section{ACKNOWLEDGEMENTS}

We are grateful for the thorough reviews by Hagay Amit and another anonymous reviewer. In addition, we thank Kathy Whaler for her constructive remarks. M. C. Metman is supported by a studentship awarded as part of the Leeds-York NERC Doctoral Training Partnership (NE/L002574/1) and by the BGS University Funding Initiative PhD studentship (S305). P. W. Livermore was partially supported by the Natural Environment Research Council grant NE/G0140431. 


\section{REFER E N CES}

Amit, H., 2014. Can downwelling at the top of the Earth's core be detected in the geomagnetic secular variation? Phys. Earth planet. Inter., 229, $110-121$.

Amit, H. \& Christensen, U.R., 2008. Accounting for magnetic diffusion in core flow inversions from geomagnetic secular variation, Geophys. J. Int., 175(3), 913-924.

Aubert, J., 2012. Flow throughout the Earth's core inverted from geomagnetic observations and numerical dynamo models, Geophys. J. Int., 192(2), 537-556.

Aubert, J., 2014. Earth's core internal dynamics 1840-2010 imaged by inverse geodynamo modelling, Geophys. J. Int., 197(3), 1321-1334.

Aubert, J., Aurnou, J. \& Wicht, J., 2008. The magnetic structure of convection-driven numerical dynamos, Geophys. J. Int., 172(3), 945-956.

Aubert, J., Finlay, C.C. \& Fournier, A., 2013. Bottom-up control of geomagnetic secular variation by the Earth's inner core, Nature, 502, 219-223.

Backus, G., 1958. A class of self-sustaining dissipative spherical dynamos, Ann. Phys., 4(4), 372-447.

Backus, G. \& Bullard, E.C., 1968. Kinematics of geomagnetic secular variation in a perfectly conducting core, Phil. Trans. R. Soc. Lond., A, 263(1141), 239-266.

Bärenzung, J., Holschneider, M., Wicht, J., Sanchez, S. \& Lesur, V., 2018. Modeling and predicting the short-term evolution of the geomagnetic field, J. geophys. Res., 123(6), 4539-4560.

Barrois, O., Gillet, N. \& Aubert, J., 2017. Contributions to the geomagnetic secular variation from a reanalysis of core surface dynamics, Geophys. J. Int., 211(1), 50-68.

Barrois, O., Gillet, N., Aubert, J., Barrois, O., Hammer, M.D., Finlay, C.C., Martin, Y. \& Gillet, N., 2018. Erratum: Contributions to the geomagnetic secular variation from a reanalysis of core surface dynamics and Assimilation of ground and satellite magnetic measurements: inference of core surface magnetic and velocity field changes, Geophys. J. Int., 216(3), 2106-2113.

Beggan, C.D. \& Whaler, K.A., 2009. Forecasting change of the magnetic field using core surface flows and ensemble Kalman filtering, Geophys. Res. Lett., 36(18), doi:10.1029/2009GL039927.

Beggan, C.D. \& Whaler, K.A., 2018. Ensemble Kalman filter analysis of magnetic field models during the CHAMP-Swarm gap, Phys. Earth planet. Inter., 281, 103-110.

Biggin, A.J., Piispa, E.J., Pesonen, L.J., Holme, R., Paterson, G.A., Veikkolainen, T. \& Tauxe, L., 2015. Palaeomagnetic field intensity variations suggest Mesoproterozoic inner-core nucleation, Nature, 526, $245-248$.

Bloxham, J., 1986. The expulsion of magnetic flux from the Earth's core, Geophys. J. R. astr. Soc., 87(2), 669-678.

Bloxham, J., 1988. The dynamical regime of fluid flow at the core surface, Geophys. Res. Lett., 15(6), 585-588.

Boyd, J.P., 2000. Chebyshev and Fourier Spectral Methods, 2ndedn, Dover Publications.

Buffett, B., 2010. Tidal dissipation and the strength of the earths internal magnetic field, Nature, 468, 952-954.

Buffett, B., 2014. Geomagnetic fluctuations reveal stable stratification at the top of the Earth's core, Nature, 507, 484-487.

Bullard, E.C., Freeman, C., Gellman, H. \& Nixon, J., 1950. The westward drift of the Earth's magnetic field, Phil. Trans. R. Soc. Lond., A, 243(859), 67-92.

Chen, L., Herreman, W., Li, K., Livermore, P.W., Luo, J.W. \& Jackson, A., 2018. The optimal kinematic dynamo driven by steady flows in a sphere, J. Fluid Mech., 839, 1-32.

Christensen, U.R., Aubert, J. \& Hulot, G., 2010. Conditions for Earth-like geodynamo models, Earth planet. Sci. Lett., 296(3), 487-496.

Chulliat, A. \& Olsen, N., 2010. Observation of magnetic diffusion in the Earth's outer core from Magsat, Ørsted, and CHAMP data, J. geophys. Res., 115(B5), doi:10.1029/2009JB006994.

Chulliat, A., Hulot, G. \& Newitt, L.R., 2010. Magnetic flux expulsion from the core as a possible cause of the unusually large acceleration of the north magnetic pole during the 1990s, J. geophys. Res., 115(B7), B007143, doi: 10.1029/2009JB007143.
Constable, C.G., Parker, R.L. \& Stark, P.B., 1993. Geomagnetic field models incorporating frozen-flux constraints, Geophys. J. Int., 113(2), 419-433.

Finlay, C.C. \& Amit, H., 2011. On flow magnitude and field-flow alignment at Earth's core surface, Geophys. J. Int., 186(1), 175-192.

Finlay, C.C. \& Jackson, A., 2003. Equatorially dominated magnetic field change at the surface of earth's core, Science, 300(5628), 2084-2086.

Fournier, A., Eymin, C. \& Alboussière, T., 2007. A case for variational geomagnetic data assimilation: insights from a one-dimensional, nonlinear, and sparsely observed mhd system, Nonlinear Process. Geophys., 14(2), $163-180$.

Gillet, N., Jault, D., Canet, E. \& Fournier, A., 2010. Fast torsional waves and strong magnetic field within the earths core, Nature, 465, 74-77.

Gillet, N., Barrois, O. \& Finlay, C.C., 2015a. Stochastic forecasting of the geomagnetic field from the COV-OBS.x1 geomagnetic field model, and candidate models for IGRF-12, Earth Planets Space, 67(1), 71.

Gillet, N., Jault, D. \& Finlay, C.C., 2015b. Planetary gyre, time-dependent eddies, torsional waves, and equatorial jets at the earth's core surface, $J$. geophys. Res., 120(6), 3991-4013.

Glatzmaier, G. \& Coe, R.S., 2015. Magnetic polarity reversals in the core, in Treatise on Geophysics, vol. 8, Elsevier, 2ndedn.

Gubbins, D., 1984. Geomagnetic field analysis - II. Secular variation consistent with a perfectly conducting core, Geophys. J. R. astr. Soc., 77(3), 753-766.

Gubbins, D., 1987. Mechanism for geomagnetic polarity reversals, Nature, 326, $167-169$.

Gubbins, D., 1996. A formalism for the inversion of geomagnetic data for core motions with diffusion, Phys. Earth planet. Inter., 98(3), 193-206.

Gubbins, D., 2007. Geomagnetic constraints on stratification at the top of Earth's core, Earth Planets Space, 59(7), 661-664.

Gubbins, D. \& Bloxham, J., 1985. Geomagnetic field analysis III. Magnetic fields on the coremantle boundary, Geophys. J. Int., 80(3), 695-713.

Gubbins, D. \& Kelly, P., 1996. A difficulty with using the frozen flux hypothesis to find steady core motions, Geophys. Res. Lett., 23(14), 1825-1828.

Gubbins, D. \& Roberts, P.H., 1987. Magnetohydrodynamics of the Earth's Core, in Geomagnetism, vol. 2, Academic Press.

Halley, E., 1692. An account of the cause of the change of the variation of the magnetical needle. with an hypothesis of the structure of the internal parts of the earth: as it was proposed to the royal society in one of their late meetings, Philos. Trans., 17(195), 563-578.

Hide, R., 1966. Free hydromagnetic oscillations of the earth's core and the theory of the geomagnetic secular variation, Phil. Trans. R. Soc. Lond., A, 259(1107), 615-647.

Holme, R., 2015. Large-scale flow in the core, in Treatise on Geophysics, vol. 8, pp. 91-113, Elsevier.

Huguet, L., Amit, H. \& Alboussire, T., 2018. Geomagnetic dipole changes and upwelling/downwelling at the top of the Earth's core, Front. Earth Sci., 6, 170 .

Jackson, A. \& Finlay, C., 2015. Geomagnetic secular variation and its applications to the core, in Treatise on Geophysics, vol. 5, Elsevier, Oxford, 2ndedn.

Jackson, A., Bloxham, J. \& Gubbins, D., 1993. Time-dependent flow at the core surface and conservation of angular momentum in the coupled coremantle system, in Dynamics of Earth's Deep Interior and Earth Rotation, pp. 97-107, American Geophysical Union (AGU).

Jault, D., Gire, C. \& Mouël, J.L.L., 1988. Westward drift, core motions and exchanges of angular momentum between core and mantle, Nature, 333, 353-356.

Lay, T. \& Young, C.J., 1990. The stably-stratified outermost core revisited, Geophys. Res. Lett., 17(11), 2001-2004.

Li, K., Jackson, A. \& Livermore, P.W., 2014. Variational data assimilation for a forced, inertia-free magnetohydrodynamic dynamo model, Geophys. J. Int., 199(3), 1662-1676.

Li, K., Jackson, A. \& Livermore, P.W., 2018. Taylor state dynamos found by optimal control: axisymmetric examples, J. Fluid Mech., 853, 647-697.

Livermore, P.W., 2010. Galerkin orthogonal polynomials, J. Comput. Phys., 229(6), 2046-2060.

Livermore, P.W. \& Jackson, A., 2006. Transient magnetic energy growth in spherical stationary flows, Proc. R. Soc. Lond., A, 462(2072), 2457-2479. 
Livermore, P.W., Hollerbach, R. \& Finlay, C.C., 2017. An accelerating highlatitude jet in earths core, Nature Geosci., 10, 62-68.

Love, J.J., 1999. A critique of frozen-flux inverse modelling of a nearly steady geodynamo, Geophys. J. Int., 138(2), 353-365.

Mandea, M. \& Dormy, E., 2003. Asymmetric behavior of magnetic dip poles, Earth Planets Space, 55(3), 153-157.

Mandea, M. \& Olsen, N., 2006. A new approach to directly determine the secular variation from magnetic satellite observations, Geophys. Res. Lett., 33(15), doi:10.1029/2006GL026616.

Mandea, M., Bellanger, E. \& Mouël, J.L.L., 2000. A geomagnetic jerk for the end of the 20th century? Earth planet. Sci. Lett., 183(3), 369-373.

Metman, M., Livermore, P. \& Mound, J., 2018. The reversed and normal flux contributions to axial dipole decay for 1880-2015, Phys. Earth planet. Inter, 276, 106-117.

Miranker, W.L., 1961. A well posed problem for the backward heat equation, Proc. Am. Math. Soc., 12(2), 243-247.

Olsen, N. \& Mandea, M., 2008. Rapidly changing flows in the Earth's core, Nature Geosci., 1, 390-394.

Olson, P. \& Amit, H., 2006. Changes in earth's dipole, Naturwissenschaften, 93(11), 519-542.

Roberts, P.H. \& Glatzmaier, G.A., 2000. A test of the frozen-flux approximation using a new geodynamo model, Phil. Trans. R. Soc. Lond., A, 358(1768), 1109-1121.
Roberts, P.H. \& Scott, S., 1965. On analysis of the secular variation, $J$. Geomag. Geoelectr., 17(2), 137-151.

Tarduno, J.A. et al., 2010. Geodynamo, solar wind, and magnetopause 3.4 to 3.45 billion years ago, Science, 327(5970), $1238-1240$

Terra-Nova, F., Amit, H., Hartmann, G.A. \& Trindade, R.I.F., 2015. The time dependence of reversed archeomagnetic flux patches, J. geophys. Res., 120(2), 691-704.

Terra-Nova, F., Amit, H., Hartmann, G.A. \& Trindade, R.I., 2016. Using archaeomagnetic field models to constrain the physics of the core: robustness and preferred locations of reversed flux patches, Geophys. J. Int., 206(3), 1890-1913.

Vestine, E.H., Ball, R.H. \& Kahle, A.B., 1967. Nature of surface flow in the Earth's central core, J. geophys. Res., 72(19), 4927-4936.

Wardinski, I. \& Lesur, V., 2012. An extended version of the C3FM geomagnetic field model: application of a continuous frozen-flux constraint, Geophys. J. Int., 189(3), 1409-1429.

Whaler, K., 1980. Does the whole of the Earth's core convect? Nature, 287, $528-530$.

Whaler, K.A. \& Beggan, C.D., 2015. Derivation and use of core surface flows for forecasting secular variation, J. geophys. Res., 120(3), 1400-1414.

\section{SUPPORTING INFORMATION}

Supplementary data are available at $G J I$ online.

\section{1_damping_parameter_picks.dat}

Please note: Oxford University Press is not responsible for the content or functionality of any supporting materials supplied by the authors. Any queries (other than missing material) should be directed to the corresponding author for the paper.

\section{APPENDIX A: DEPTH SENSITIVITY OF SV OBSERVATIONS}

Here we present our means to determine what region of the core our model of purely diffusive SV is sensitive to given data on the CMB. We do this by quantifying the diffusive signature on the CMB of delta-function structures at various depths. We use the decay modes presented in Section 2.1 and define with these functions the initial magnetic anomaly

$r s_{\alpha}\left(r, t_{0}\right)=\delta\left(r-r_{0}\right) \simeq \sum_{n=1}^{N} q_{\alpha}^{n} r d_{l_{\alpha}}^{n}\left(r, t_{0}\right)$,

where $\delta(x)$ is the Dirac delta function centred at $r_{0} \in(0, c)$. To find the associated coefficients $q_{\alpha}^{n}$ we project onto the decay modes using the functional inner product

$\langle f, g\rangle_{l_{\alpha}}:=\frac{4 \pi l_{\alpha}\left(l_{\alpha}+1\right)}{2 l_{\alpha}+1}\left[\int_{0}^{c}\left(\frac{\partial f}{\partial r}\right)\left(\frac{\partial g}{\partial r}\right)+\frac{l_{\alpha}\left(l_{\alpha}+1\right)}{r^{2}} f g \mathrm{~d} r+\frac{l_{\alpha}}{c} f(c) g(c)\right]$,

which for $f=g=r s_{\alpha}$ corresponds to the magnetic energy over all space defined by $s_{\alpha}$ (e.g. Li et al. 2018; see also Appendix D). The projection then yields the coefficients

$q_{\alpha}^{n}=\frac{\left\langle\delta\left(r-r_{0}\right), r d_{l_{\alpha}}^{n}\left(r, t_{0}\right)\right\rangle_{l_{\alpha}}}{\left\langle r d_{l_{\alpha}}^{n}\left(r, t_{0}\right), r d_{l_{\alpha}}^{n}\left(r, t_{0}\right)\right\rangle_{l_{\alpha}}}=\frac{2 r_{0} d_{l_{\alpha}}^{n}\left(r_{0}, t_{0}\right)}{c^{3} j_{l_{\alpha}+1}^{2}\left(k_{l_{\alpha}}^{n}\right)}$.

With these coefficients, we may compute for all $l_{\alpha}$ a diffusive response $\left[r s_{\alpha}(c, t)\right]_{t_{0}}^{t_{1}}$ that is the amplitude of the anomaly at the CMB after $t_{1}$ $-t_{0}$ years of diffusion. To determine how sensitive CMB observations are to different depths inside the core, we vary the radius $r_{0}$ at which the initial anomaly is centred.

Fig. A1 shows the amplitude of three different resultant anomalies for each $l_{\alpha} \in\{1,8,14\}$ after $175 \mathrm{yr}$ of diffusion (matching the full COV-OBS.x1 period), which are centred at $r_{0} \in\{c / 4, c / 2,0.95 c\}$. These have been computed with $N=200$, which ensures numerical convergence, as integration over $[0, c]$ of the decay mode representation of the delta function is within one percent of unity. While all anomalies assume similar curvature, the decay of these features is notably faster for higher degrees, with this separation being more pronounced at greater depth. Additionally, the anomalies at $0.95 \mathrm{c}$ are spatially less symmetric compared to those at greater depth due to the imposed matching condition at $r=c$ (Gubbins \& Roberts 1987, eq. 3.56). This figure confirms that the diffusive evolution of deep magnetic structures 


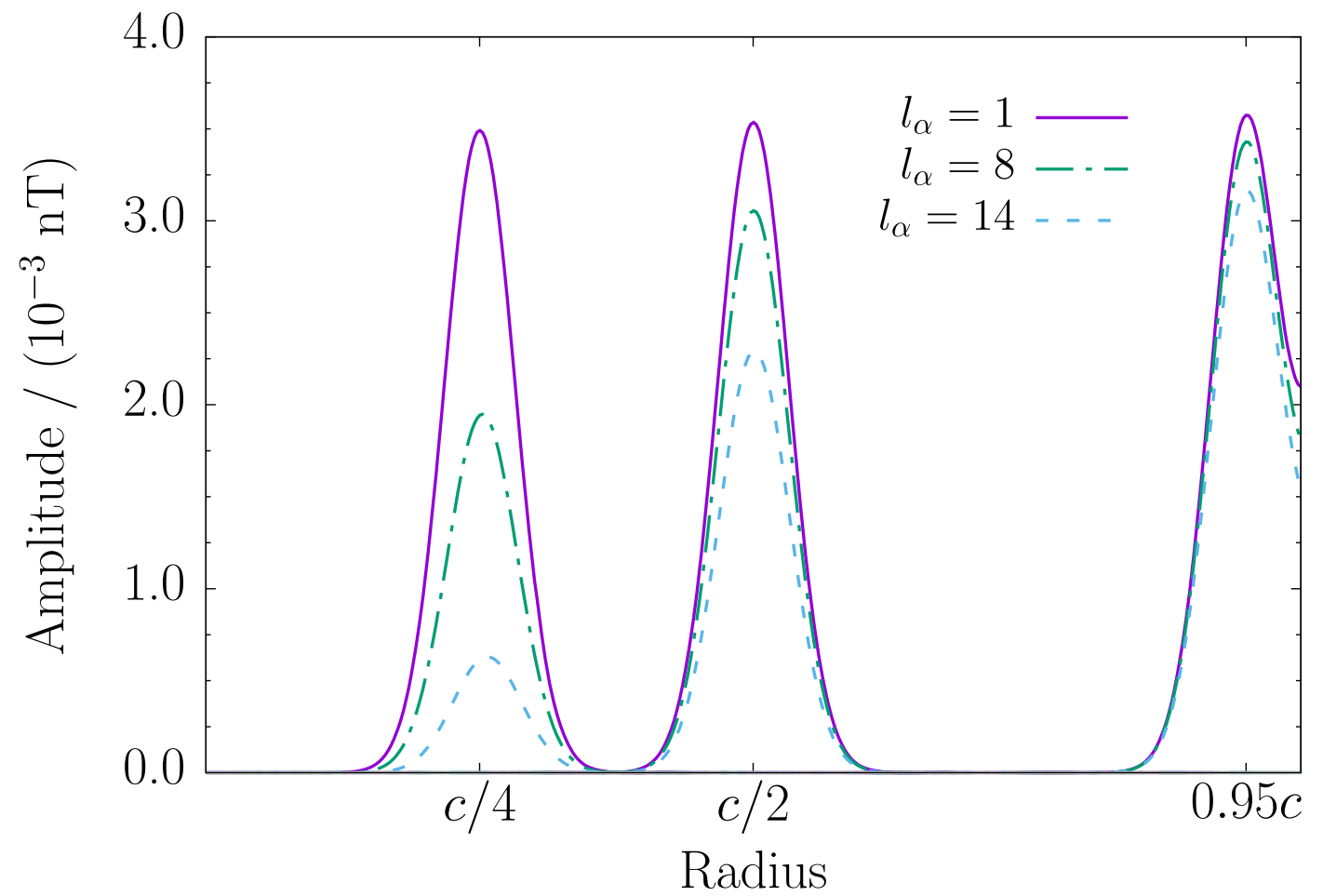

Figure A1. Amplitude of the degree 1, 8 and 14 magnetic anomalies, centred at three different depths: $(c / 4, c / 2,0.95 c)$ after $175 \mathrm{yr}$ of diffusion.

is unobservable at the CMB after $175 \mathrm{yr}$, since the amplitude of the relatively deep $r_{0}=c / 4$ anomaly is negligible beyond $c / 2$. Conversely, the diffusion of shallow features, such as the $r_{0}=0.95$ anomaly, has a clear signature at the CMB.

Fig. A2 highlights this depth sensitivity in more detail by showing for all degrees the diffusive response at the CMB as a function of the central radius $r_{0}$ for 10 and $175 \mathrm{yr}$ of diffusion ( $\mathrm{a}$ and $\mathrm{b}$, respectively). In both figures, the response is weaker for features of increasing degree, and this discrepancy is more striking when the time window is lengthened. Most importantly, these response functions demonstrate that for purely diffusive the CMB field evolution can only constrain the magnetic structure within the uppermost core, that is the upper $80 \mathrm{~km}$ when one decade of diffusion is considered (Fig. A2a); for $175 \mathrm{yr}$ of diffusion the CMB field evolution is sensitive to roughly the upper $400 \mathrm{~km}$ (Fig. A2b). 

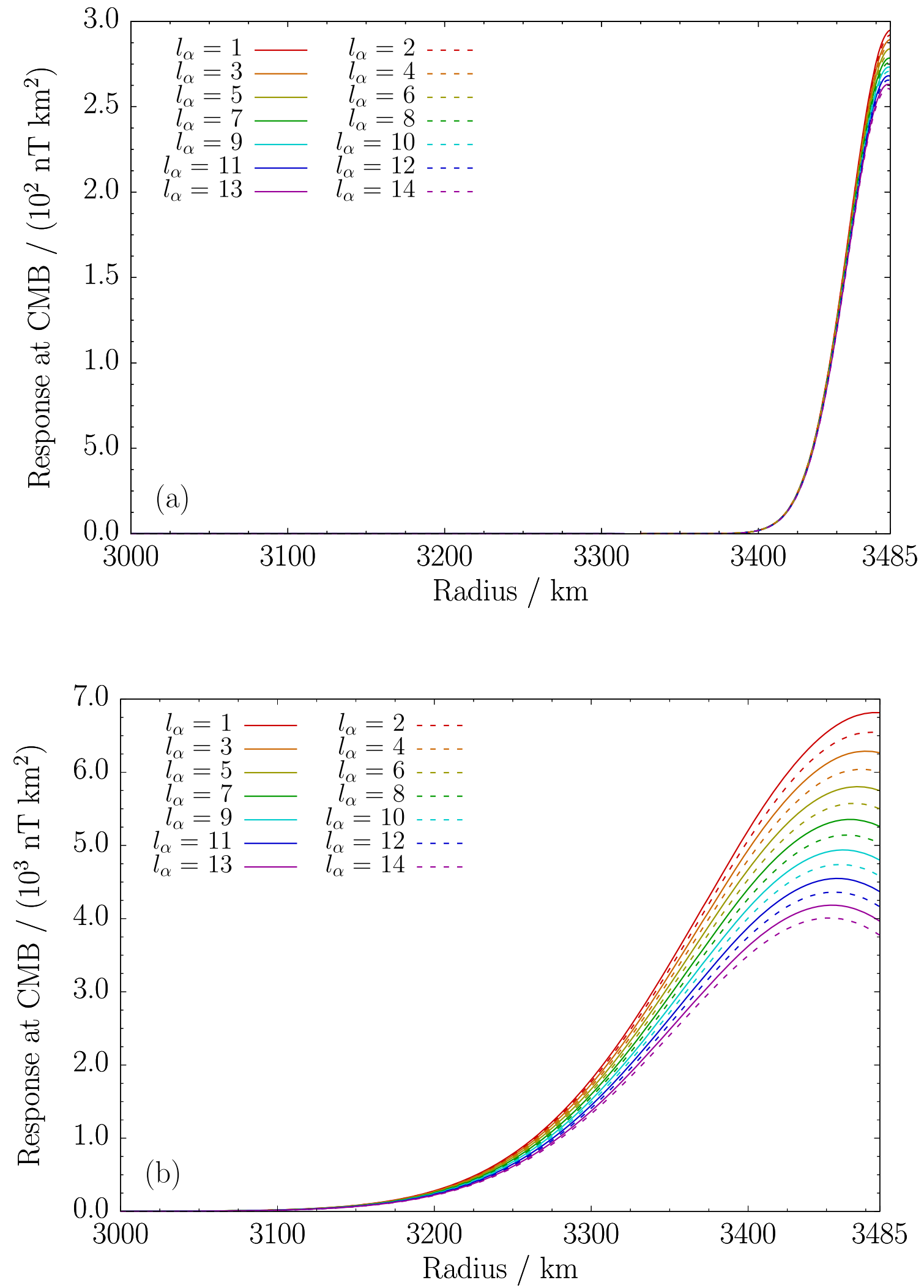

Figure A2. The diffusive response at the CMB of the magnetic impulse anomaly as a function of the radius at which the anomaly is centred, after $10 \mathrm{yr}$ (a) and $175 \mathrm{yr}(\mathrm{b})$. 


\section{APPENDIX B: DERIVATION OF THE DIFFUSIVE LEAST-SQUARES RESIDUAL}

In this paper, there are two representations of the magnetic field. The first is the poloidal field defined within the electrically conducting core (e.g. Gubbins \& Roberts 1987), and the second is geomagnetic field model defined as a potential field. Respectively these are given by

$B_{r}(\mathbf{r}, t)=\left\{\begin{array}{ll}\frac{1}{r} \sum_{\alpha=1}^{L(L+2)} l_{\alpha}\left(l_{\alpha}+1\right) s_{\alpha}(r, t) Y_{\alpha}(\theta, \phi), & r \leq c \\ \sum_{\alpha=1}^{L(L+2)}\left(l_{\alpha}+1\right)\left(\frac{a}{r}\right)^{l_{\alpha}+2} g_{\alpha}(t) Y_{\alpha}(\theta, \phi), & r \geq c\end{array}\right.$.

Constructing the residual on $r=c$ then leads to

$\left.\left(B_{r}-\hat{B}_{r}\right)\right|_{r=c}=\sum_{\alpha=1}^{L(L+2)}\left(l_{\alpha}+1\right)\left(\frac{a}{c}\right)^{l_{\alpha}+2}\left[g_{\alpha}(t)-\frac{l_{\alpha}}{c}\left(\frac{c}{a}\right)^{l_{\alpha}+2} \hat{s}_{\alpha}(c, t)\right] Y_{\alpha}(\theta, \phi)$,

where the hat denotes our modelled field. It then follows from the orthogonality of the spherical harmonics that

$\int_{\mathrm{CMB}}\left(B_{r}-\hat{B}_{r}\right)^{2} \mathrm{~d} \Omega=4 \pi c^{2} \sum_{\alpha=1}^{L(L+2)} \frac{\left(l_{\alpha}+1\right)^{2}}{2 l_{\alpha}+1}\left(\frac{a}{c}\right)^{2 l_{\alpha}+4}\left[g_{\alpha}(t)-\frac{l_{\alpha}}{c}\left(\frac{c}{a}\right)^{l_{\alpha}+2} \hat{s}_{\alpha}(c, t)\right]^{2}$.

We may substitute the Galerkin approximation for the poloidal coefficients (eq. 15), and write the coefficients $g_{\alpha}\left(t_{k}\right)$ and $\hat{s}_{\alpha}\left(t_{k}\right)$ as $g_{\alpha, k}$ and $\hat{s}_{\alpha, k}$, respectively. Time integration of this residual then yields

$\int_{T} \int_{\mathrm{CMB}}\left(B_{r}-\hat{B}_{r}\right)^{2} \mathrm{~d} \Omega \simeq 4 \pi c^{2} \sum_{\alpha=1}^{L(L+2)} \frac{\left(l_{\alpha}+1\right)^{2}}{2 l_{\alpha}+1}\left(\frac{a}{c}\right)^{2 l_{\alpha}+4}\left(\mathbf{g}_{\alpha}-\mathbf{D}_{\alpha} \hat{\mathbf{q}}_{\alpha}\right)^{T} \mathbf{T}\left(\mathbf{g}_{\alpha}-\mathbf{D}_{\alpha} \hat{\mathbf{q}}_{\alpha}\right)$,

where $D_{\alpha, i j}=\frac{l_{\alpha}}{c^{2}}\left(\frac{c}{a}\right)^{l_{\alpha}+2}\left(\xi_{\alpha}(c)^{T} \operatorname{expm}\left[\mathbf{H}_{\alpha}\left(t_{i}-t_{0}\right)\right]\right)_{j}$ and $\mathbf{T}$ a diagonal weighting matrix corresponding to the numerical temporal integration scheme used. Here we use Simpson's rule, in which case:

$T_{i j}=\left\{\begin{array}{cl}\delta t / 3 \delta_{i j} & i=1 \text { or } i=K \\ 4 \delta t / 3 \delta_{i j} & i \text { even and } 1<i<K \\ 2 \delta t / 3 \delta_{i j} & i \text { odd and } 1<i<K\end{array}\right.$

with $\delta t$ the interval length between time points and $K$ the number of time points. If we define the quantities: $\mathbf{q}=\left(\mathbf{q}_{1}, \mathbf{q}_{2}, \ldots, \mathbf{q}_{L(L+2)}\right)^{T}, \mathbf{g}=$ $\left(\mathbf{g}_{1}, \mathbf{g}_{2}, \ldots, \mathbf{g}_{L(L+2)}\right)^{T}, \mathbf{D}=\operatorname{diag}\left(\mathbf{D}_{1}, \mathbf{D}_{2}, \ldots, \mathbf{D}_{L(L+2)}\right), \mathbf{W}_{\alpha}=4 \pi c^{2} \frac{\left(l_{\alpha}+1\right)^{2}}{2 l_{\alpha}+1}\left(\frac{a}{c}\right)^{2 l_{\alpha}+4} \mathbf{T}$ and $\mathbf{W}=\operatorname{diag}\left(\mathbf{W}_{1}, \mathbf{W}_{2}, \ldots, \mathbf{W}_{L(L+2)}\right)$ we may further simplify the residual and show that it corresponds to a weighted least-squares problem:

$\int_{T} \int_{\mathrm{CMB}}\left(B_{r}-\hat{B}_{r}\right)^{2} \mathrm{~d} \Omega \mathrm{d} t=(\mathbf{g}-\mathbf{D q})^{T} \mathbf{W}(\mathbf{g}-\mathbf{D q})$

with the solution minimizing this residual:

$\hat{\mathbf{q}}=\left(\mathbf{D}^{T} \mathbf{W D}\right)^{-1} \mathbf{D}^{T} \mathbf{W g}$

\section{APPENDIX C: NUMERICAL CONVERGENCE}

The least-squares solution (eq. 18) relies on several approximations, namely the use of a finite radial truncation $N$ and a discretized numerical time integration with $K$ abscissae: here we use Simpson's rule. We test what values of $N$ and $K$ are needed for an accurate and numerically converged solution.

First, consider Fig. C1 showing a normalized objective function $R_{\text {unreg }}$, that is the RMS error in $B_{\mathrm{r}}$, against the number of basis functions $N$ used for the inversion. These solutions have been obtained using sextuple (256-digit) precision and the mean COV-OBS.x 1 field model (Gillet et al. 2015a) over the period 1840-2015, with either $K=103$ or $K=203$ time points (purple crosses and green circles, respectively). It can be seen that the residual decreases monotonically with $N$; for $N>21$ there is a regime in which this decay is more gradual. As such, increasing $N$ beyond 30 can be expected to return a much reduced increase in accuracy-the relative difference between the $N=29$ and $N=$ 30 residuals is already only about 1 per cent. We therefore consider the solution to be sufficiently converged for $N=30$. For this choice of $N$, the figure also shows that $K=103$ and $K=203$ produce almost the same result.

Second, Fig. C2 shows the normalized $R_{\text {unreg }}$ as a function of the number of time points used, acquired with the same field model and floating-point precision as for Fig. C1, and for either 2 or 30 basis functions (purple crosses and green circles, respectively). The rate of convergence appears rather sensitive to the number of basis functions, as a converged solution for $N=30$ requires significantly more time points compared to the $N=2$ case. However, for both choices the relative change in the residual when increasing $K$ beyond 203 is less than 1 per cent, so we consider this number of time points to be sufficient.

The requirements in terms of truncation parameters for both $N$ and $K$ follow similarly for the regularized cases. 


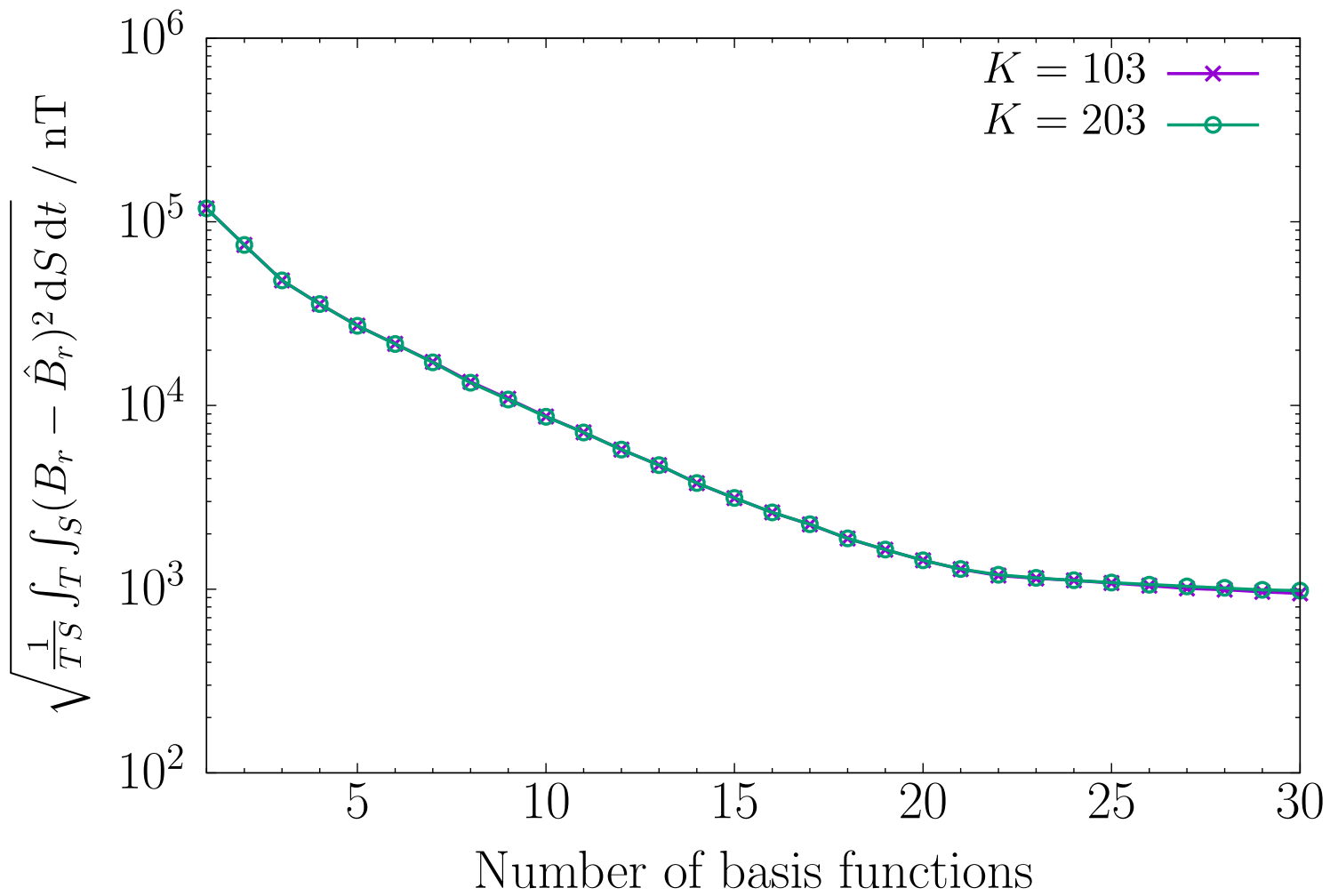

Figure C1. Dependence of the unregularized objective function $R_{\text {unreg }}$, normalized to represent an RMS residual in space and time, on the number of basis functions used for the inversion. The purple crosses and green circles correspond to the use of 103 or 203 time points, respectively.

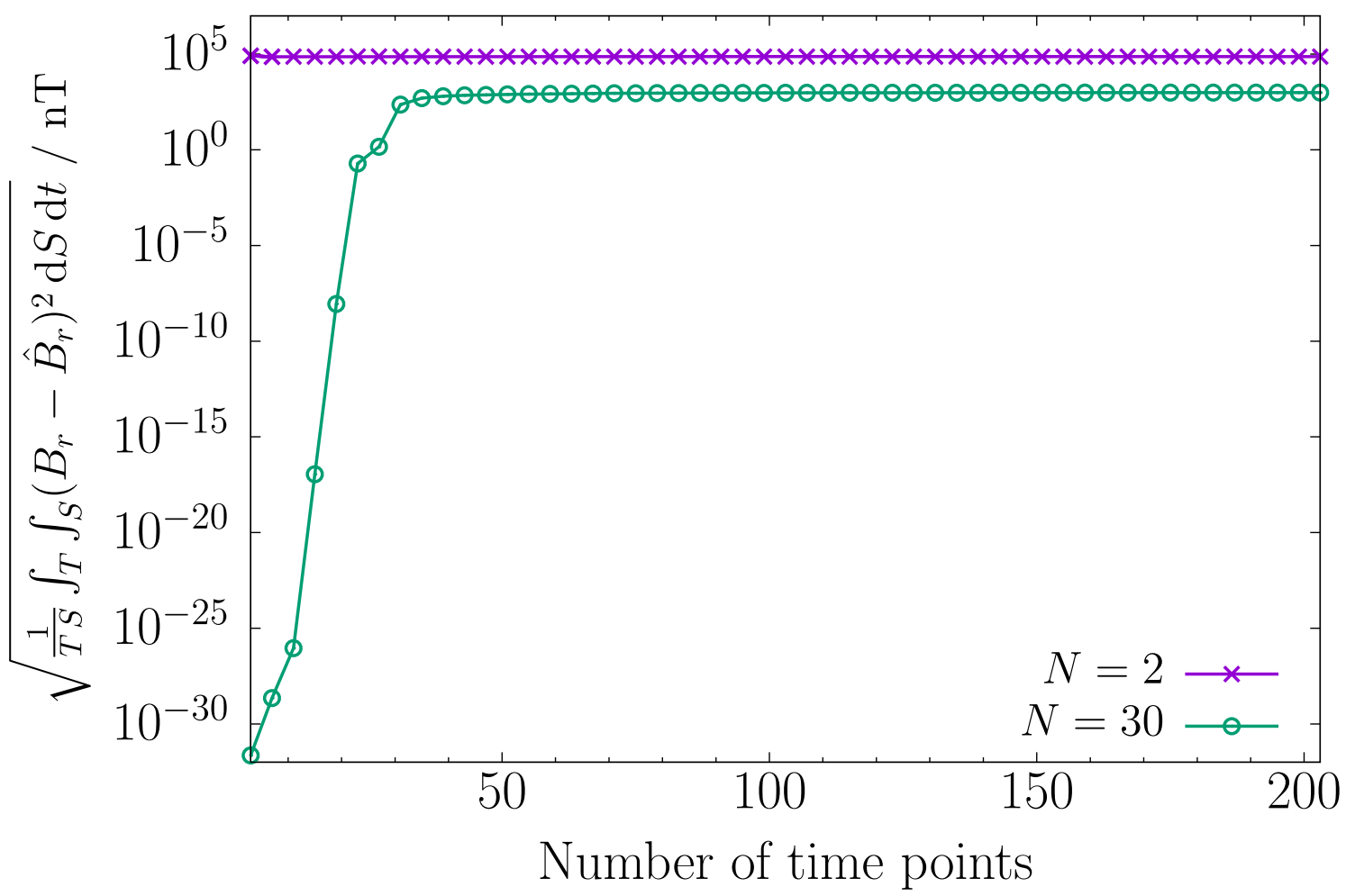

Figure C2. Dependence of the unregularized objective function $R_{\text {unreg }}$, normalized to represent an RMS residual in space and time, on the number of time points used for the inversion. The purple crossed and green circles correspond to the use of 2 or 30 basis functions, respectively. 
APPENDIX D: DERIVATION OF THE MAGNETIC ENERGY REGULARIZATION TERM

To incorporate the energy term into our inverse problem, we need to express it in terms of our model coefficients. To do so we first partition all magnetic energy into internal and external parts:

$\int_{R^{3}} \hat{\mathbf{B}}_{0}^{2} \mathrm{~d} V=\int_{V_{\text {int }}} \hat{\mathbf{B}}_{0}^{2} \mathrm{~d} V+\int_{V_{\text {ext }}} \hat{\mathbf{B}}_{0}^{2} \mathrm{~d} V$

where $V_{\text {int }}$ is the core volume and $V_{\text {ext }}$ is the space outside the core. The external field is purely poloidal and fully constrained by the poloidal field inside the core:

$\mathbf{B}_{\mathrm{ext}}(\mathbf{r})=-\sum_{\alpha=1}^{L(L+2)} l_{\alpha} s_{\alpha}(c) \nabla\left[\left(\frac{c}{r}\right)^{l_{\alpha}+1} Y_{\alpha}(\theta, \phi)\right]$

Therefore, we may write

$\int_{V_{\text {ext }}} \hat{\mathbf{B}}_{0}^{2} \mathrm{~d} V=4 \pi c \sum_{\alpha=1}^{L(L+2)} \frac{l_{\alpha}^{2}\left(l_{\alpha}+1\right)}{2 l_{\alpha}+1} \hat{s}_{\alpha}^{2}(c)$,

where we have used the identity

$\int_{\Omega} \frac{\partial Y_{\alpha}}{\partial \theta} \frac{\partial Y_{\beta}}{\partial \theta}+\frac{1}{\sin ^{2} \theta} \frac{\partial Y_{\alpha}}{\partial \phi} \frac{\partial Y_{\beta}}{\partial \phi} \mathrm{d} S=\frac{4 \pi l_{\alpha}\left(l_{\alpha}+1\right)}{2 l_{\alpha}+1} \delta_{\alpha \beta}$

in which $\Omega$ is the surface of the unit sphere. Our modelled internal model field is also poloidal, and with the same identity we can derive

$\int_{V_{\text {int }}} \hat{\mathbf{B}}_{0}^{2} \mathrm{~d} V=4 \pi \sum_{\alpha=1}^{L(L+2)} \frac{l_{\alpha}\left(l_{\alpha}+1\right)}{2 l_{\alpha}+1} \int_{0}^{c} \frac{l_{\alpha}\left(l_{\alpha}+1\right)}{r^{2}}\left(r s_{\alpha}\right)^{2}+\left(\frac{\partial\left(r s_{\alpha}\right)}{\partial r}\right)^{2} \mathrm{~d} r$.

Adding the internal and external contributions we obtain

$\int_{R^{3}} \hat{\mathbf{B}}_{0}^{2}=4 \pi \sum_{\alpha=1}^{L(L+2)} \frac{l_{\alpha}\left(l_{\alpha}+1\right)}{2 l_{\alpha}+1}\left[\int_{0}^{c} \frac{l_{\alpha}\left(l_{\alpha}+1\right)}{r^{2}}\left(r s_{\alpha}\right)^{2}+\left(\frac{\partial\left(r s_{\alpha}\right)}{\partial r}\right)^{2} \mathrm{~d} r+c l_{\alpha} s_{\alpha}^{2}(c)\right]$.

We apply the Galerkin approximation $r s_{\alpha}\left(r, t_{0}\right)=\sum_{n}^{N} q_{n}^{\alpha} \xi_{n}^{\alpha}(r)$ :

$\int_{R^{3}} \hat{\mathbf{B}}_{0}^{2}=\sum_{\alpha=1}^{L(L+2)} \sum_{n=1}^{N} \sum_{j=1}^{N} q_{n}^{\alpha}\left\langle\xi_{n}^{\alpha}, \xi_{j}^{\alpha}\right\rangle_{l_{\alpha}} q_{j}^{\alpha}$

By definition $\left\langle\xi_{n}^{\alpha}, \xi_{j}^{\alpha}\right\rangle_{l_{\alpha}}=\delta_{n j}$, so that

$$
\int_{R^{3}} \hat{\mathbf{B}}_{0}^{2}=\sum_{\alpha=1}^{L(L+2)} \mathbf{q}_{\alpha}^{T} \mathbf{q}_{\alpha}=\mathbf{q}^{T} \mathbf{q} .
$$

